\title{
Applications of Robust, Radiation Hard AIGaN Optoelectronic Devices in Space Exploration \& High Energy Density Physics
}
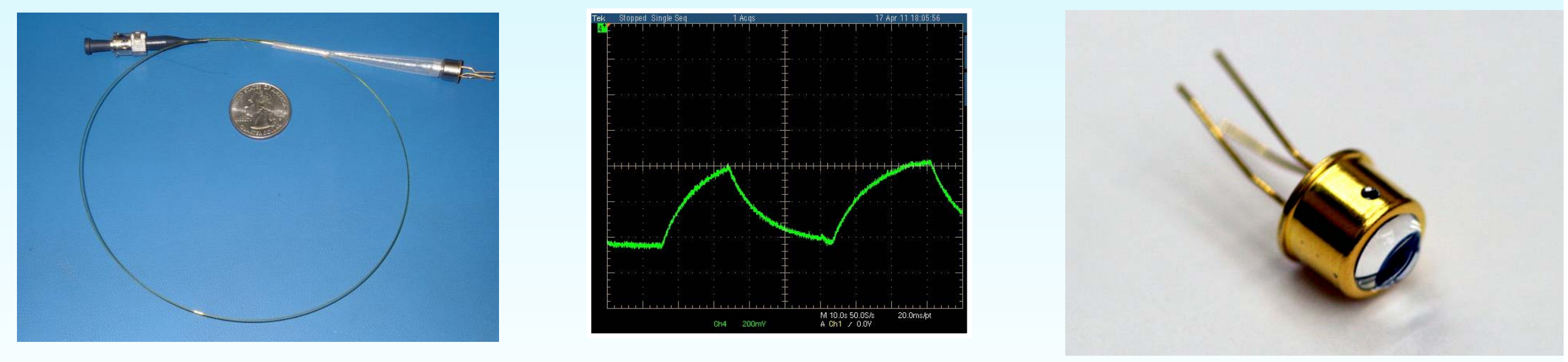

$$
\text { Ke-Xun Sun }
$$

Stanford University \& National Security Technologies

CLEO May 2011, Baltimore, Maryland 


\section{AIGaN UV LED and Photodiode Collaboration}

Sponsor I Program Management

- NASA Ames Research Ctr -- Pete Worden, BJ Jaroux

- KACST --- Turki Alsaud, Haithem Altwaijry

- Stanford University -- Robert Byer, Sasha Buchman

- National Security Technologies (DOE Contract No. DE-AC52-06NA25946-1221)

Science and Engineering Team

- Stanford University

- Ke-Xun Sun (Lead)

- Abdul Alfauwaz

- Ahmad Aljadaan

- Hamoud Aljabreen

- Muflih Alrufaydah

- Salman Althubiti

- Karthik Balakrishnan

- Eric Hultgren

- Steve Rapinchuk

- Seiya Shimizu

- Andreas Zoellner

Graduated Students:

- $\quad$ Brett Allard (AA Engr 2005)

- Sei Higuchi (AA Ph.D 2009)

- Nick Leindecker (EE MS 2008)

- Patrick Lu (EE Ph.D 2009)
- NASA Ames Research Center

- John Goebel

- John Hanson

- Kuok Ling

- Robert Ricks

- KACST

Michael Soulage

- Abdulla Alangary

- National Security Technologies (NSTec)

- Ke-Xun Sun

- Anita Behnke

- Rob Buckles

- Larry MacNeil

- William Warthan

- Sensor Electronics Technologies (SETi)

- Remis Gaska

- Yuri Bilenko

- Xuhong Hu

- Max Shatalov

- Wenhong Sun

- Jinwei Yang 


\section{Outline}

Applications of Robust, Radiation Hard AIGaN Optoelectronic Devices in Space Exploration \& High Energy Density Physics

- Space Exploration Applications

- High Energy Density Physics Applications

- UV LED and Photodiode Radiation Hardness

- UV LED and Photodiode Space Qualification

- UV LED AC Charge Management

- UV LED Satellite Payload Instruments

- UV LED Satellite will be launched $2^{\text {nd }}$ Half 2012

- Outlook 


\section{Next Generation Space Science Missions}

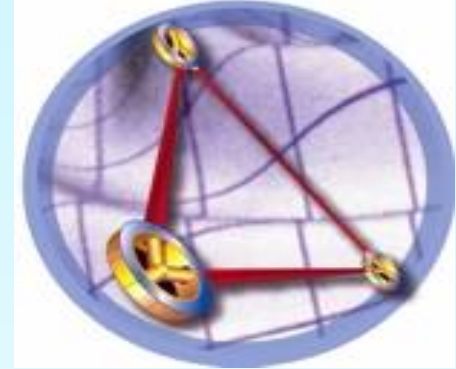

LISA

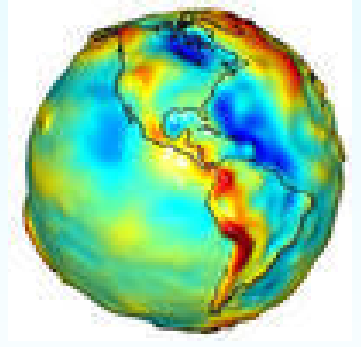

Geodesic Missions
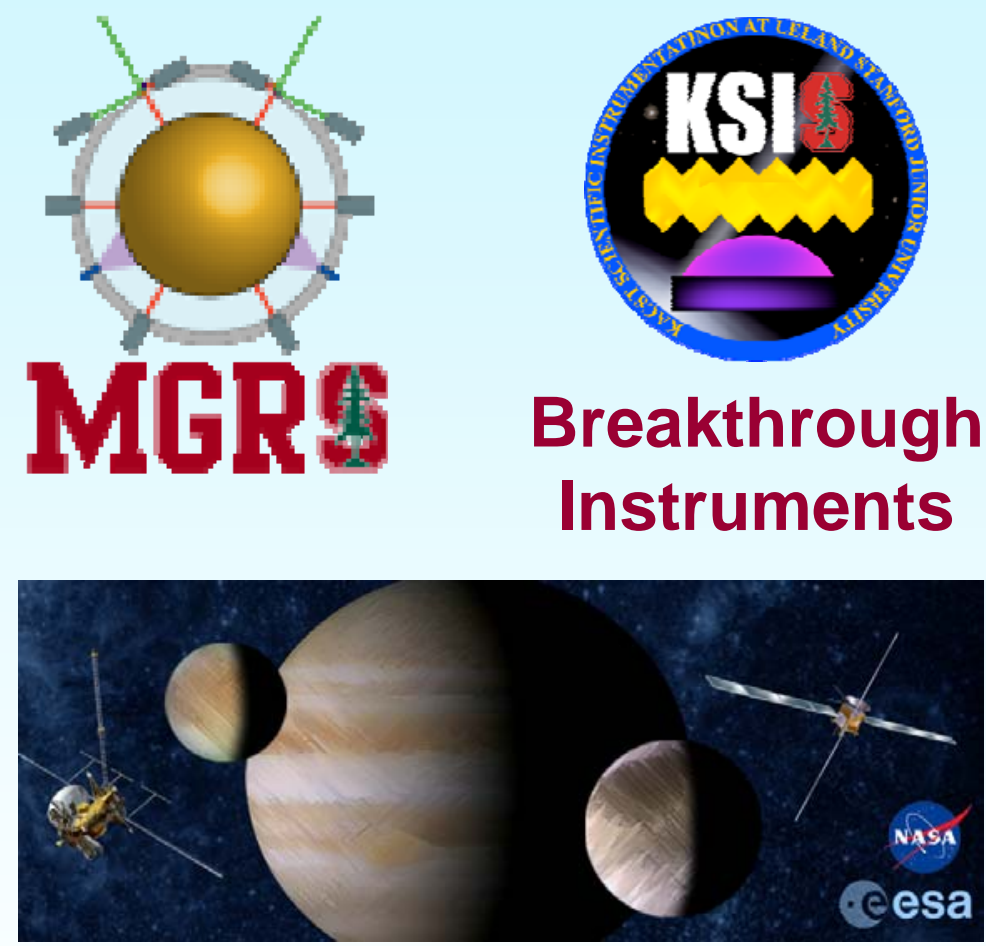

Deep Space Exploration

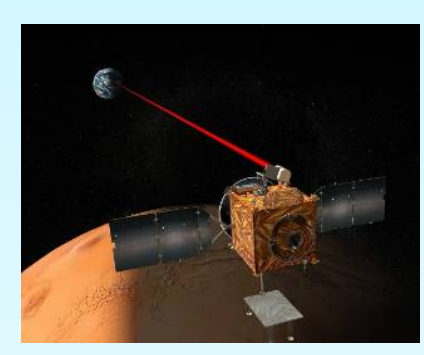

Precision Satellite Control
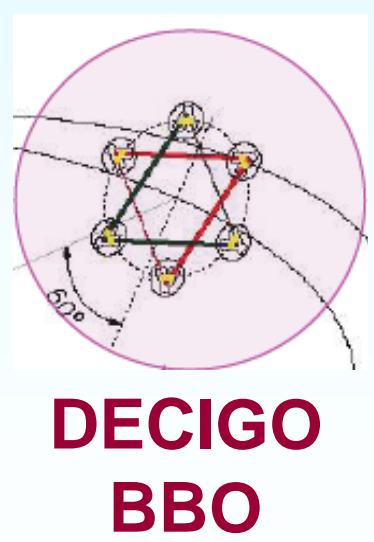


\section{Space Flight Environments and Requirements}

Radiation Environment for 3 and 7 Solar Active Years at LISA Solar Orbit

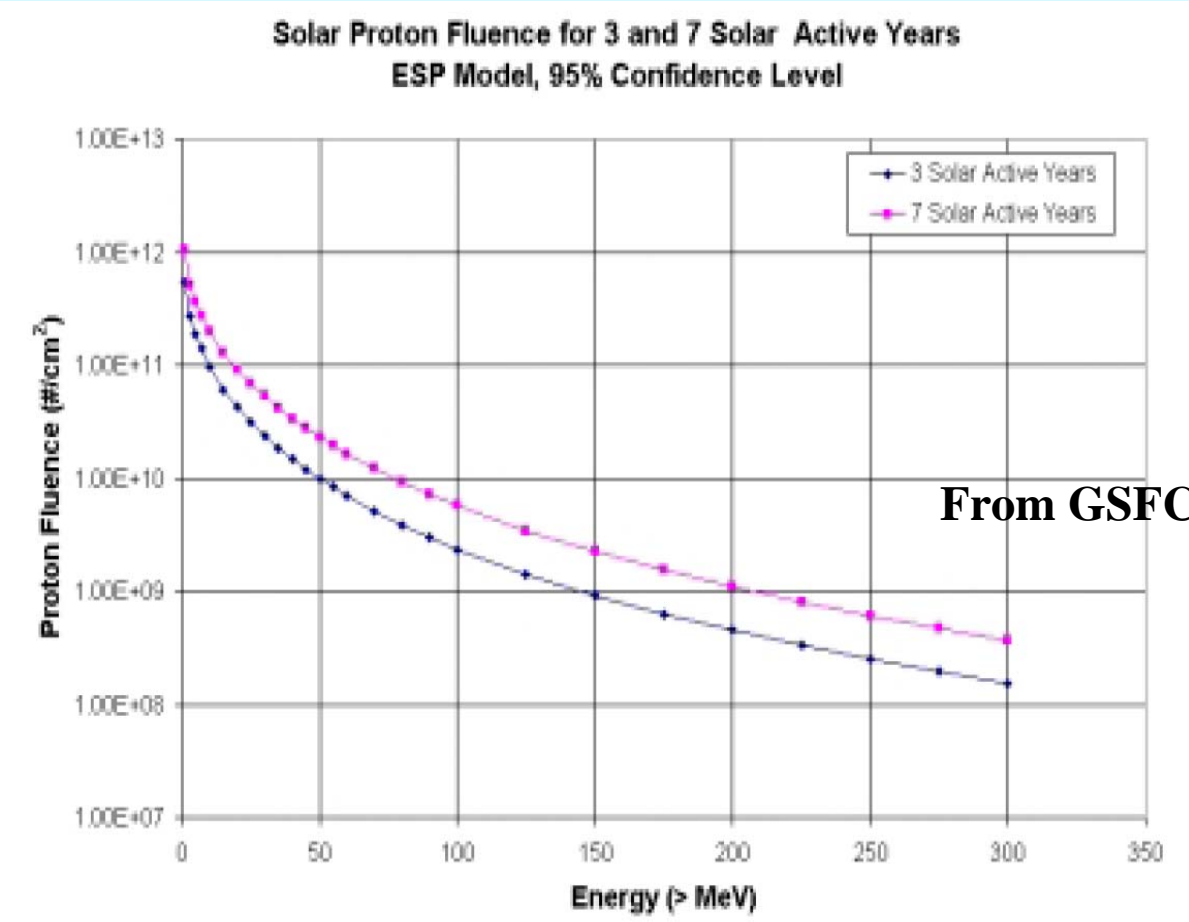

Figure 1: Solar proton fluences for a 95\% confidence level are presented for 3 and 7 solar active years.

Charging effects Radiation damages
Launch Shocks and Vibrations

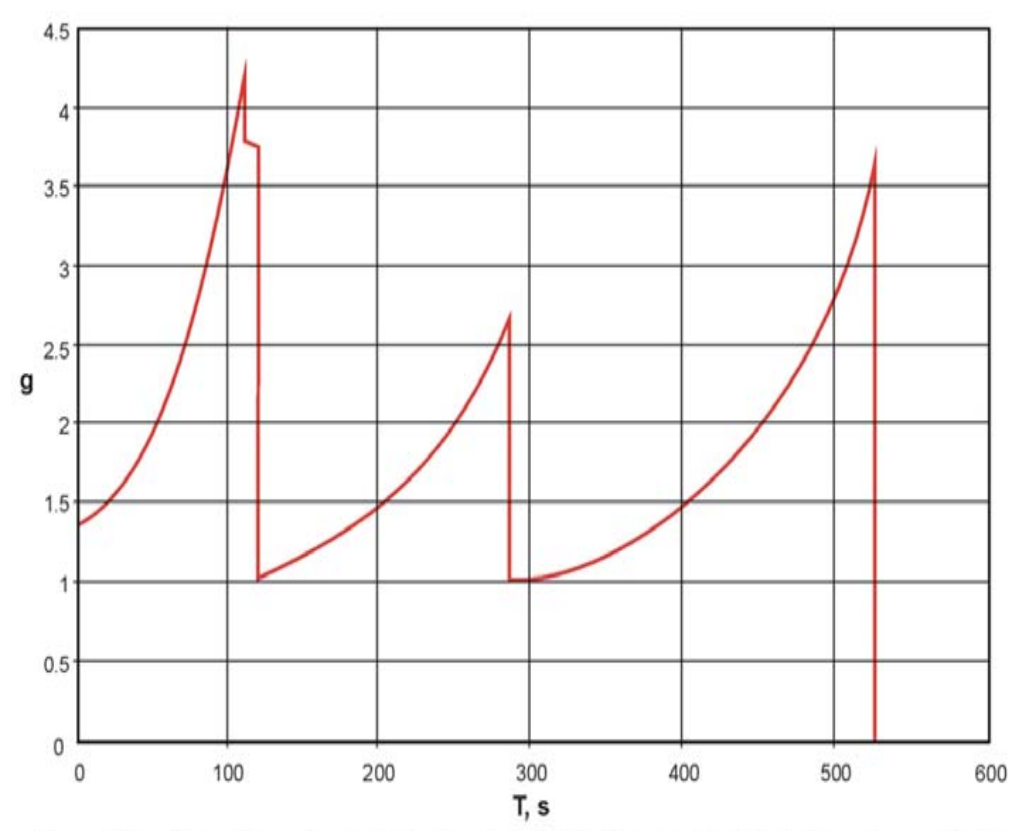

Figure 3.1 - Typical Longitudinal Steady-state Static Acceleration (first three stages flight)

Extreme Temperature Variations 


\section{Radiation Challenges for Europa Jupiter System Missions (EJSM)}

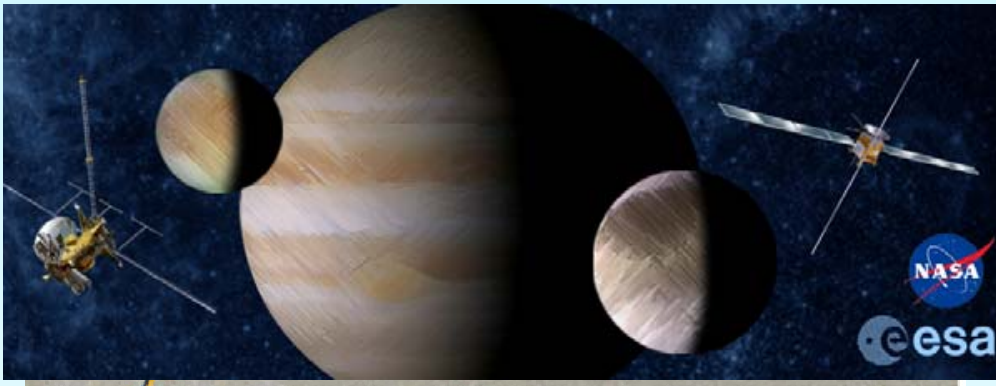

- EJSM is the flagship mission for NASA/ESA outer planet exploration programs

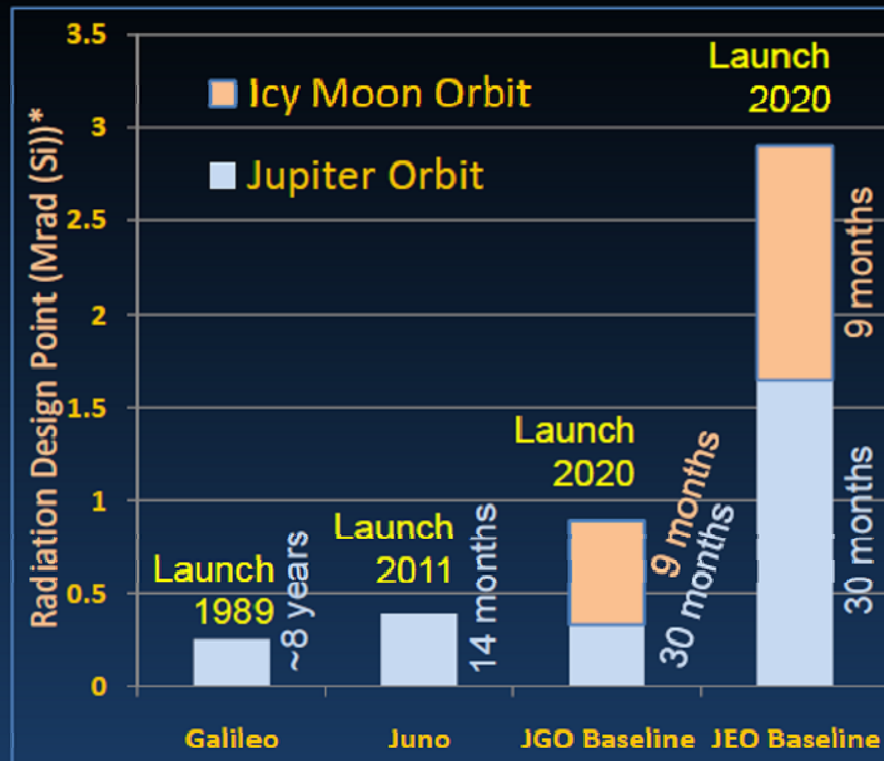

- JEO to fly over Europa

- JGO to fly over Ganymede

- total $1 \times 10^{12}$ protons/cm2 fluence expected for mission lifetime

- Radiation challenge unprecedented for NASA missions

Behind 100 mil $(2.5 \mathrm{~mm})$ of aluminum

Graph from T. Y. Yan presentation "Risk Mitigation Effort Overview" at EJSM Workshop 2009, Baltimore 


\section{NIF Neutron Fluence}

- Ignition shot: $\mathrm{N} \sim 10^{15}$ neutrons

- Neutron energy $\sim 15 \mathrm{MeV}$

- Detector $1 \mathrm{~m}$ away from target

- Neutron emission per shot $\sim 10^{15}$

$$
F=\frac{N}{4 \pi R^{2}} \approx 8 \times 10^{9} 1 / \mathrm{cm}^{2}
$$

- One year fluence (700 shots)

$F_{1 \text { year }} \approx 5.6 \times 10^{12} 1 / \mathrm{cm}^{2}$

- Proton irradiation test fluence

$F_{\text {test }} \approx(1-5) \times 10^{12} 1 / \mathrm{cm}^{2}$

- Current "Rad-Hard" films, requiring frequent replacements

- Radiation hazard for film workers

- In-efficiency

- Low performance

- Radiation hard semiconductor detectors as permanent sensors

- No radiation hazard by eliminating film operations

- Efficiency with real-time data access

- High performance

- Long term needs by LIFE and spent fuel re-use 


\section{Radiation Hardness Requirements at New Generation HEDP Facilities}

\begin{tabular}{|c|c|c|c|c|c|}
\hline Facility & $\begin{array}{c}\text { Particle \& } \\
\text { Energy }\end{array}$ & $\begin{array}{c}\text { Total \# Particles } \\
\text { Per Shot } \\
\text { (or beam } \\
\text { brightness) }\end{array}$ & $\begin{array}{c}\text { One Shot } \\
\text { Total Fluence } \\
\text { at 1 m away } \\
\left(1 / \mathrm{cm}^{2}\right)\end{array}$ & $\begin{array}{c}\text { One Year } \\
\text { Total Fluence } \\
\text { at 1 m away } \\
\left(1 / \mathrm{cm}^{2}\right)\end{array}$ & $\begin{array}{c}1 \mathrm{~mm}^{2} \\
\text { Detector } \\
\text { Total } \\
\text { Particle } \\
\text { counts }\end{array}$ \\
\hline NIF & $\begin{array}{c}\text { neutrons } \\
15 \mathrm{MeV}\end{array}$ & $10^{15}$ & $-8 \times 10^{9}$ & $\begin{array}{c}\sim 5.6 \times 10^{12} \\
(700 \text { shots })\end{array}$ & $\sim 5.6 \times 10^{10}$ \\
\hline LCLS & $\begin{array}{c}\text { X-ray } \\
8 \mathrm{keV}\end{array}$ & $10^{12}$ & $10^{12}$ & $\begin{array}{c}2 \times 10^{20} \\
(250 \text { day } \\
\text { operation) }\end{array}$ & $2 \times 10^{18}$ \\
\hline LHC & $\begin{array}{c}\text { Proton } \\
1 \mathrm{GeV}\end{array}$ & $10^{15 \sim} 10^{16}$ & $10^{15} 10^{16}$ & Higher Flux & $\begin{array}{c}\text { Higher } \\
\text { Flux }\end{array}$ \\
\hline
\end{tabular}




\section{UV LED Spectrum Measured at Stanford}

- Peak wavelength:

- FWHM:

- Total UV power:
$257.2 \mathrm{~nm}$, comparable to $\mathrm{Hg}$ line $254 \mathrm{~nm}$

$12.5 \mathrm{~nm}$, good photoemission for Au coatings

$0.144 \mathrm{~mW}$, sufficient for charge management

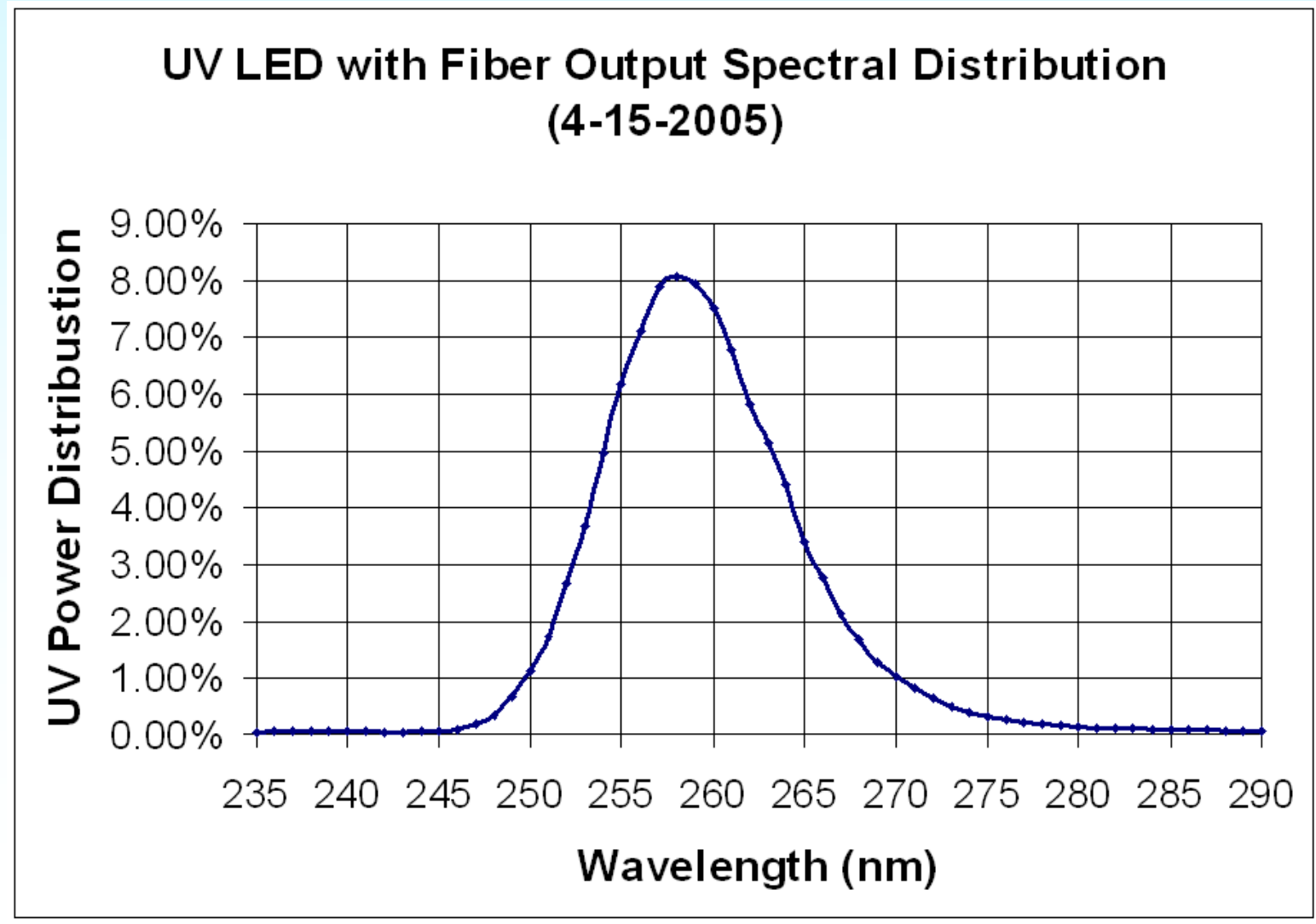




\section{UV LED Power and Spectral Stability}

\section{Lifetime tests started March 2006, over 5 years. Still Running.}

Operation Lifetime in Nitrogen $>26,000$ hours
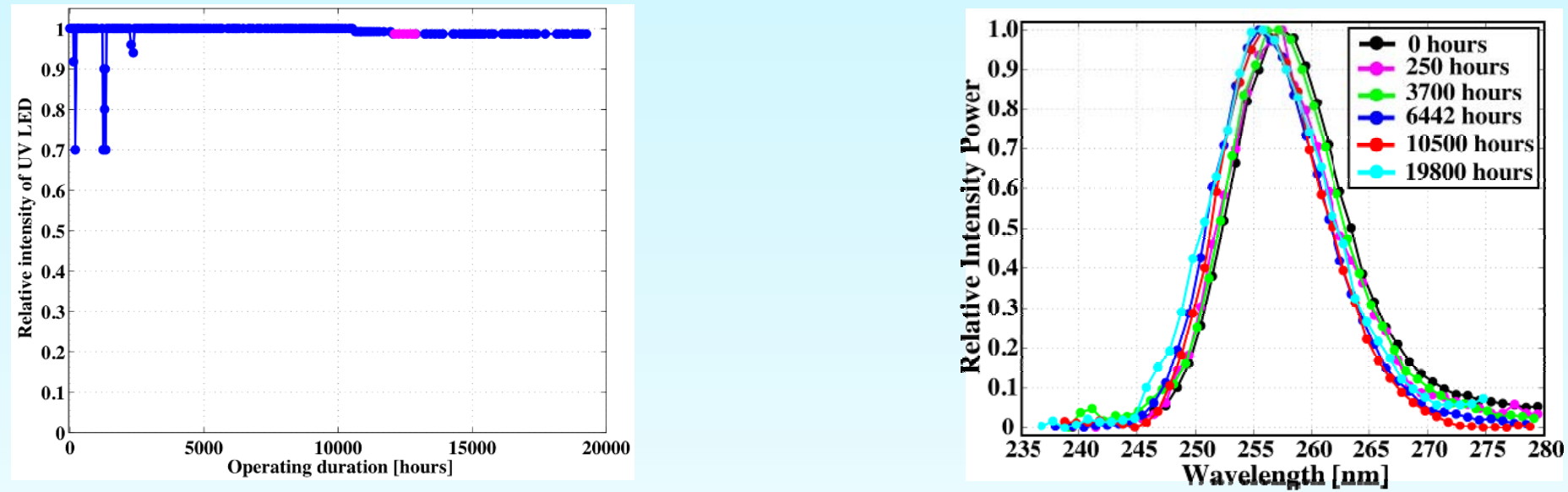

Operation Lifetime in Vacuum > 30,000 hours
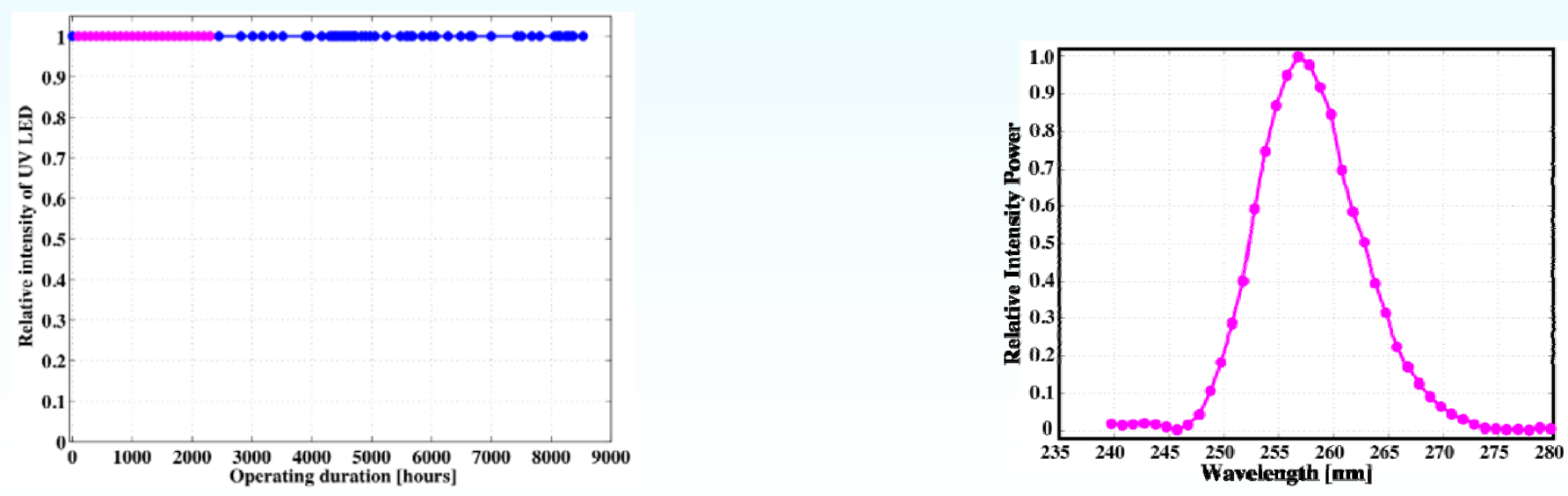

K.-X. Sun et al "UV LED Operation Lifetime and Radiation Hardness Qualification for Space Flights,” Journal of Physics CS, doi: 10.1088/1742-6596/154/1/012028 


\section{UV LED Radiation Hardness Test Proton Irradiation Setup at UC Davis}

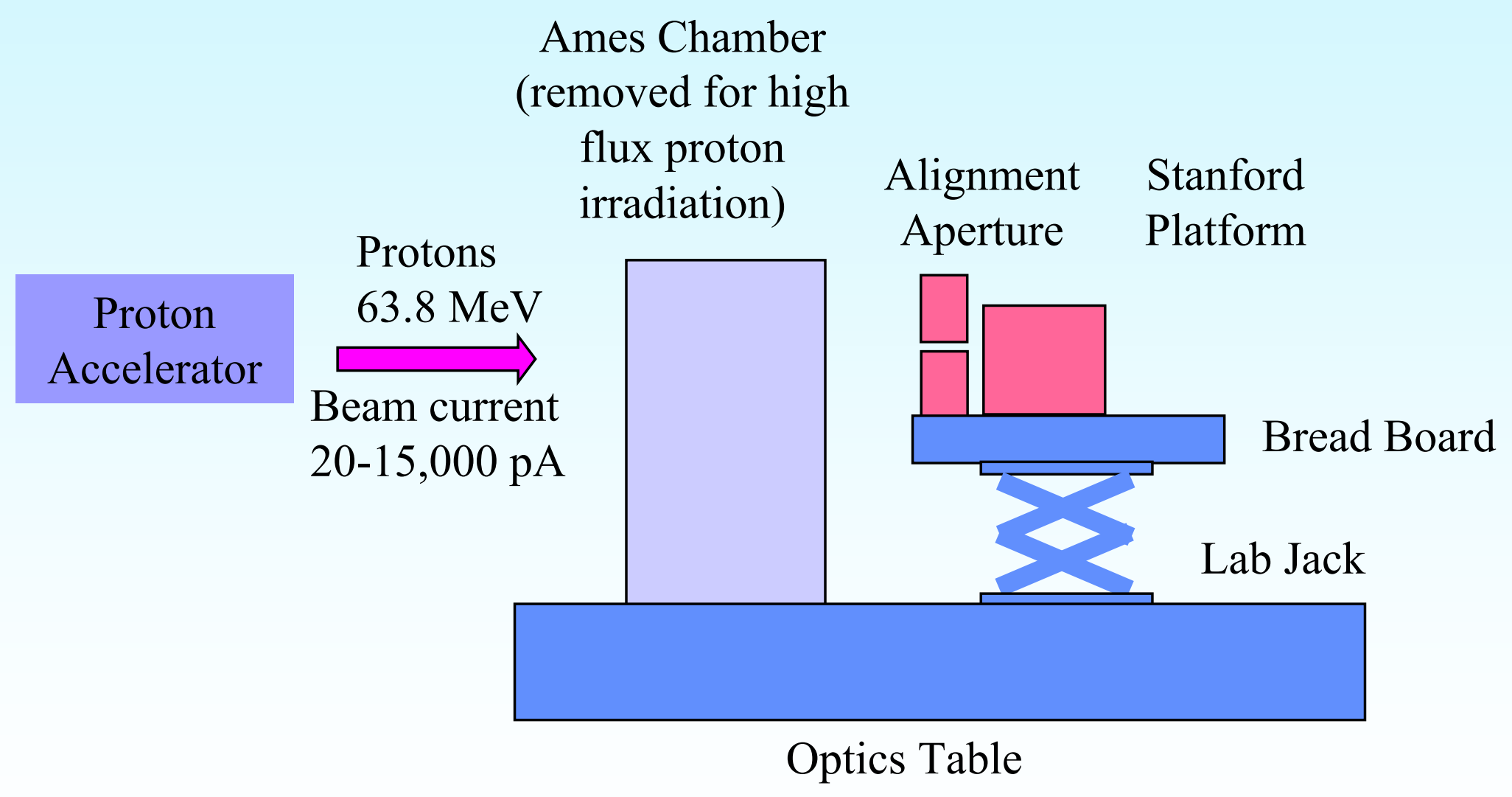




\section{UC Davis Proton Beam Facility}
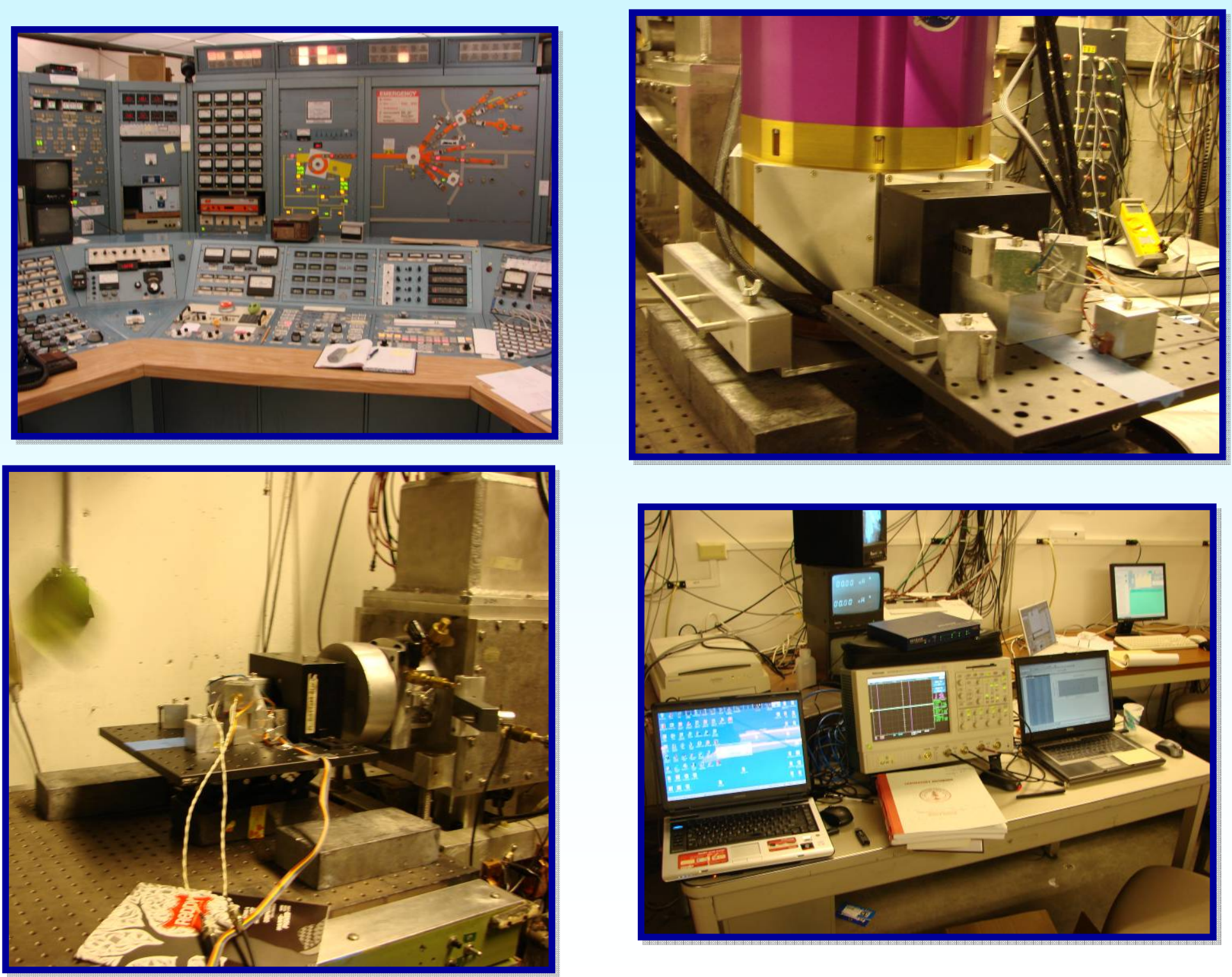

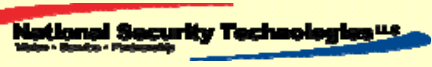

Technology, Inc. Nitrde Technology 


\section{UV LED Radiation Hardness Using Proton Irradiation}

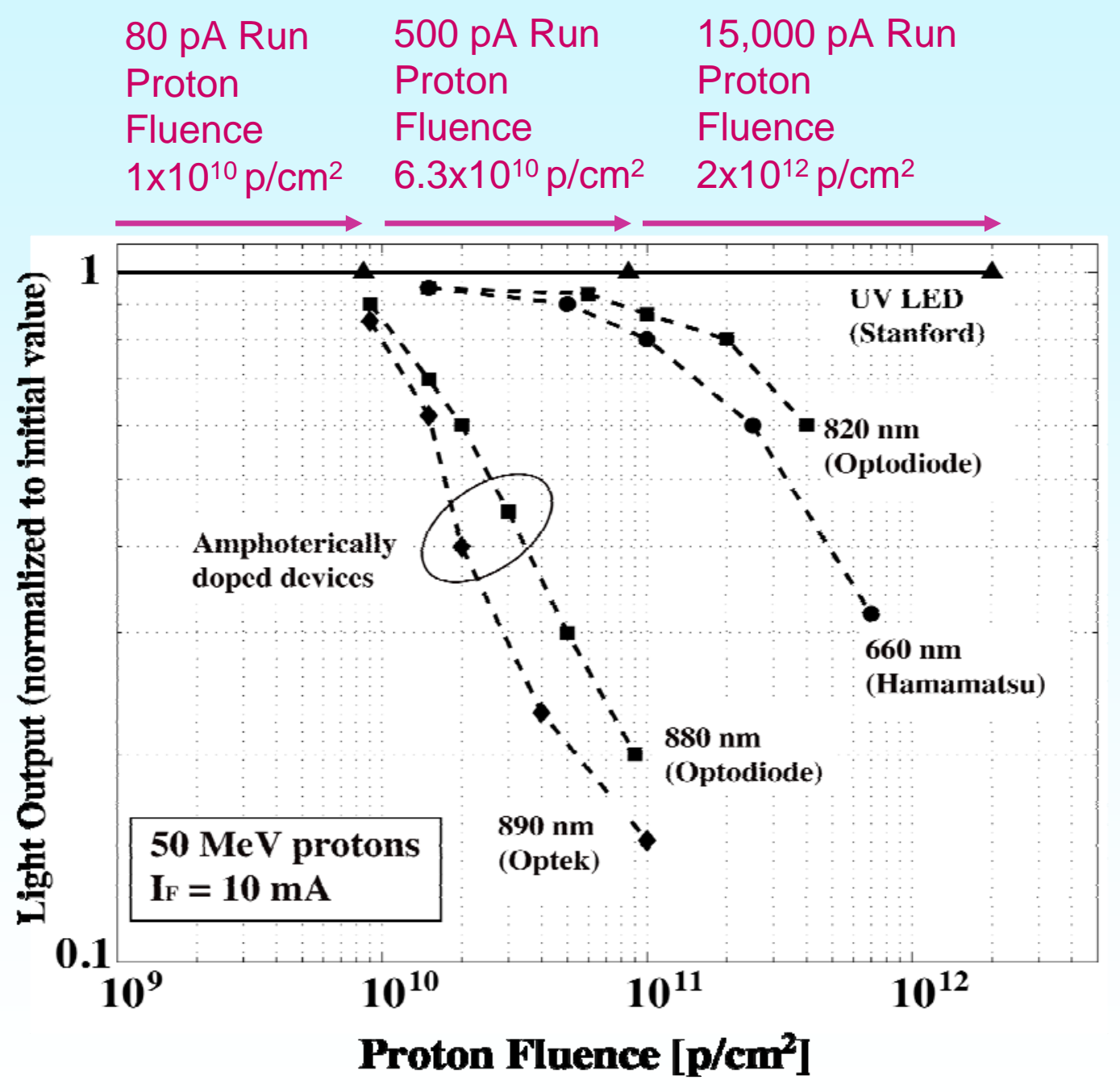

UC Davis proton energy: $59 \mathrm{MeV}$ for 80pA \& $500 \mathrm{pA}$ 63.8 MeV for $15,000 \mathrm{pA}$

Space proton energy: 2 5 MeV

Total fluence: $>100$ year proton fluence in LISA orbit

Reference for proton test of other LED and laser diodes:

A. H. Johnston and T. F. Miyahira, "Characterization of Proton Damage in Light-Emitting Diodes", IEEE Trans. Nuclear Science, 47 (6), 1999

Sun, Leindecker, et al, “UV LED Qualification for Space Flight”, 


\section{AIGaN Photodiode Proton Irradiation Test}

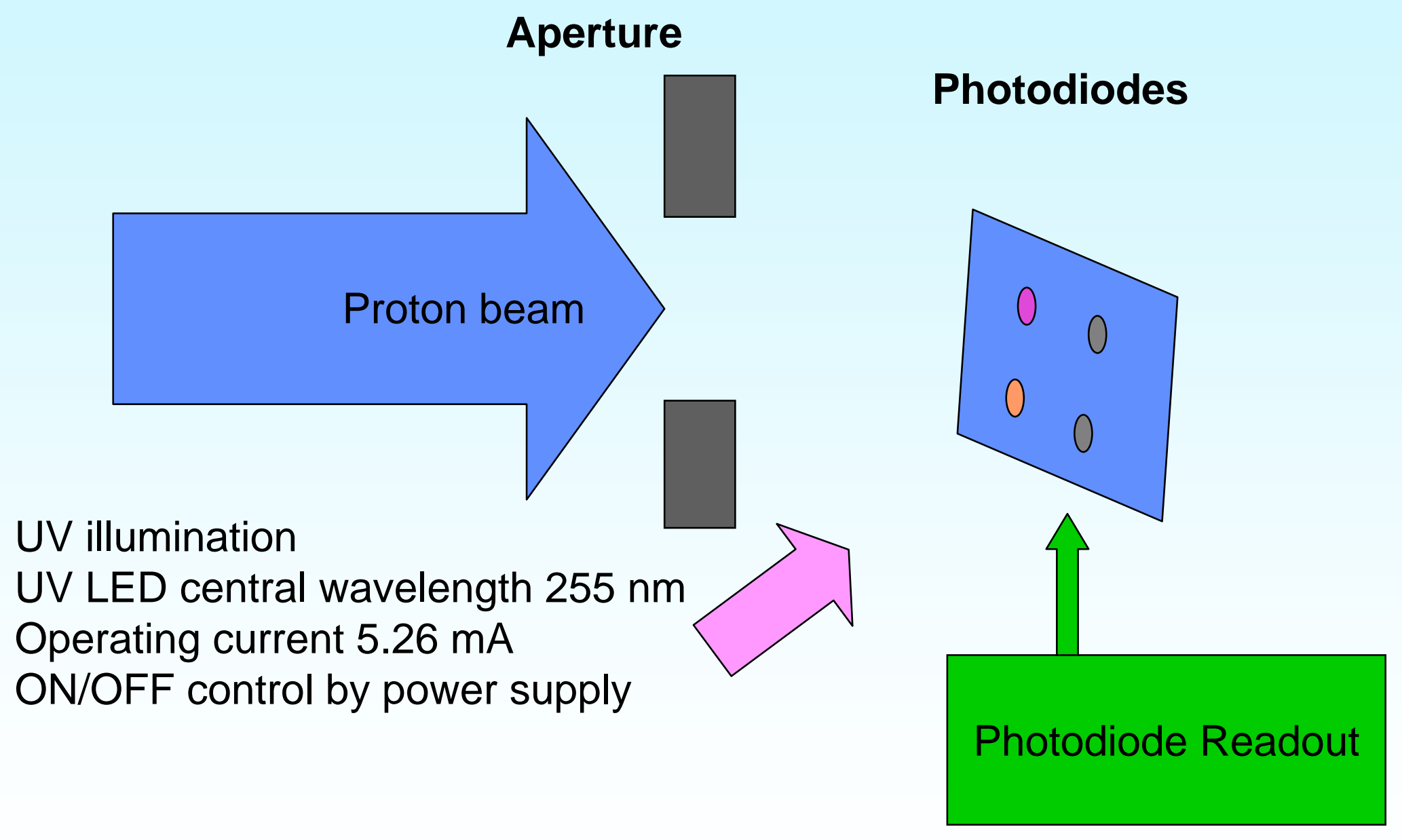

Work at National Security Technologies, LLC is done under Contract No. DE-AC52-06NA25946 with the U.S. Department of Energy with the U.S. Department of Energy. DOE/NV/25946--1221 


\section{AIGaN UV Photodiode Radiation Hardness Test UC Davis Proton Beam Facility}
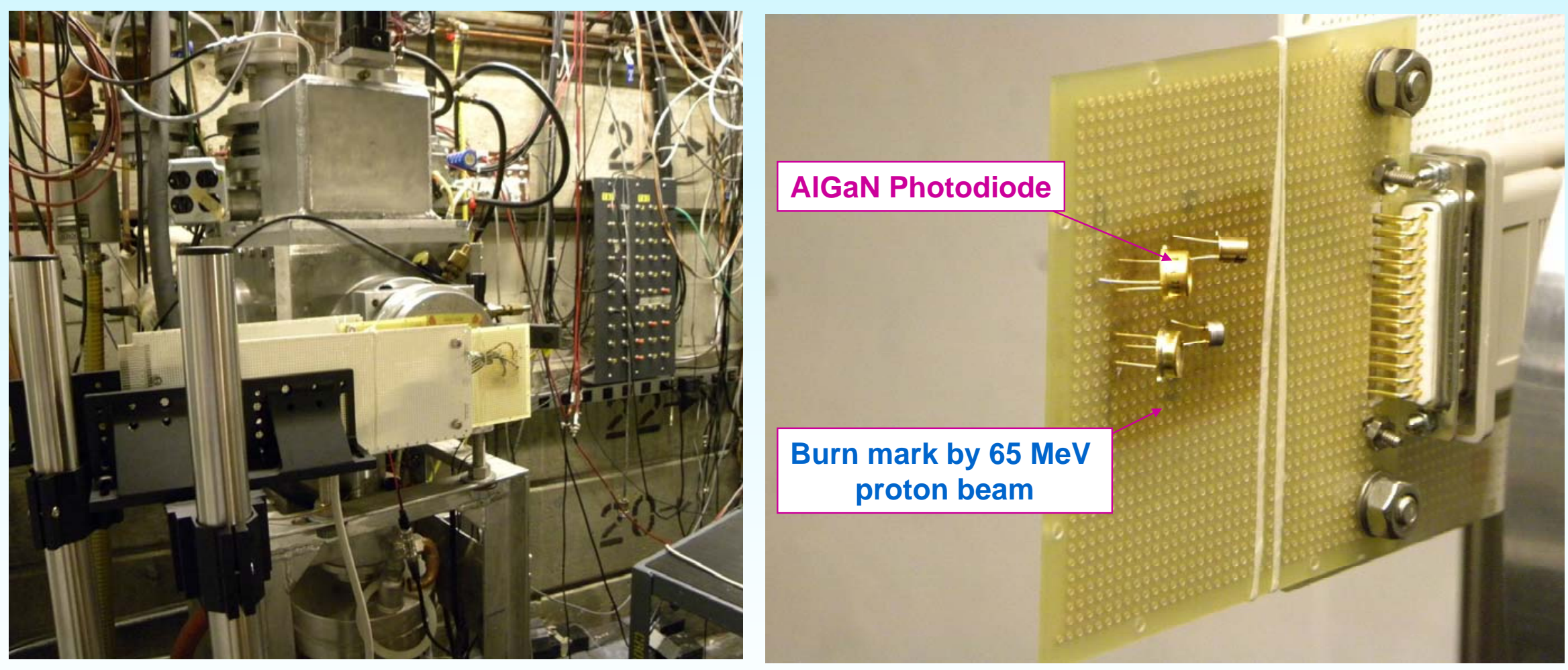

Work at National Security Technologies, LLC is done under Contract No. DE-AC52-06NA25946 with the U.S. Department of Energy with the U.S. Department of Energy. DOE/NV/25946--1221 


\section{AlGaN Photodiode UV Light Response vs. Proton Fluence}

- Normalized Response

- Photodiode in photovoltaic mode

- For each fluence level, measure the photodiode readout for UV light on and off

- The normalized differential reading is defined as the photodiode response to UV light

Work at National Security Technologies, LLC is done under Contract No. DE-AC52-06NA25946 with the U.S. Department of Energy with the U.S. Department of Energy. DOE/NV/25946--1221

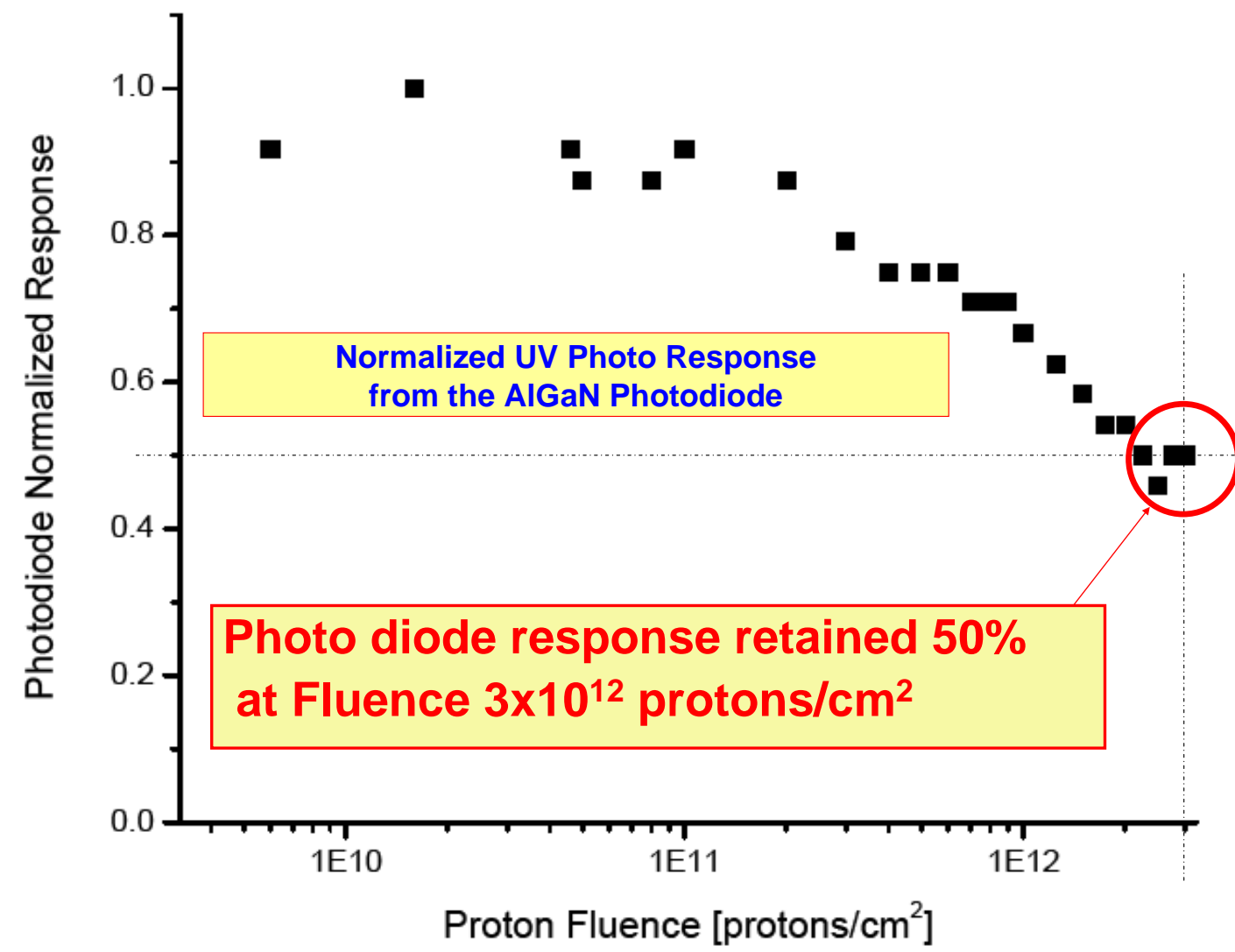

[1] K.-X. Sun and L. MacNeil, "Radiation Hardness of AlGaN Photodiodes," 4th NASA/ESA EJSM Workshop, Jet Propulsion Laboratory, July 26-29, 2010 [2] K.-X. Sun and L. MacNeil, "GaN Radiation Hard Properties and Detectors," SPIE Hard X-ray, Gamma Ray, and Neutron Detection, San Diego, August 1-5, 2010 


\section{NASA Ames Shake \& Bake Tests 2009}

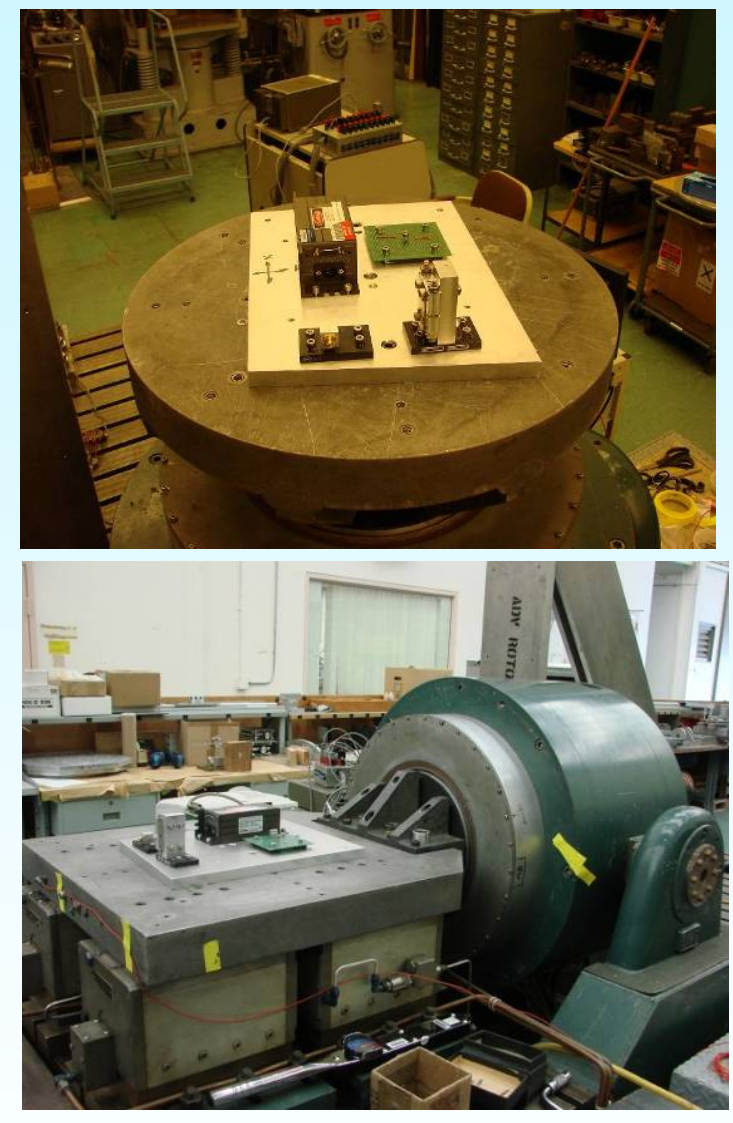

Shake Test Platform In Z-direction (upper) In $X, Y$ direction (lower) Vibration profile: $3 g$ and $7 g$ spread spectrum up to $2 \mathrm{kHz}$
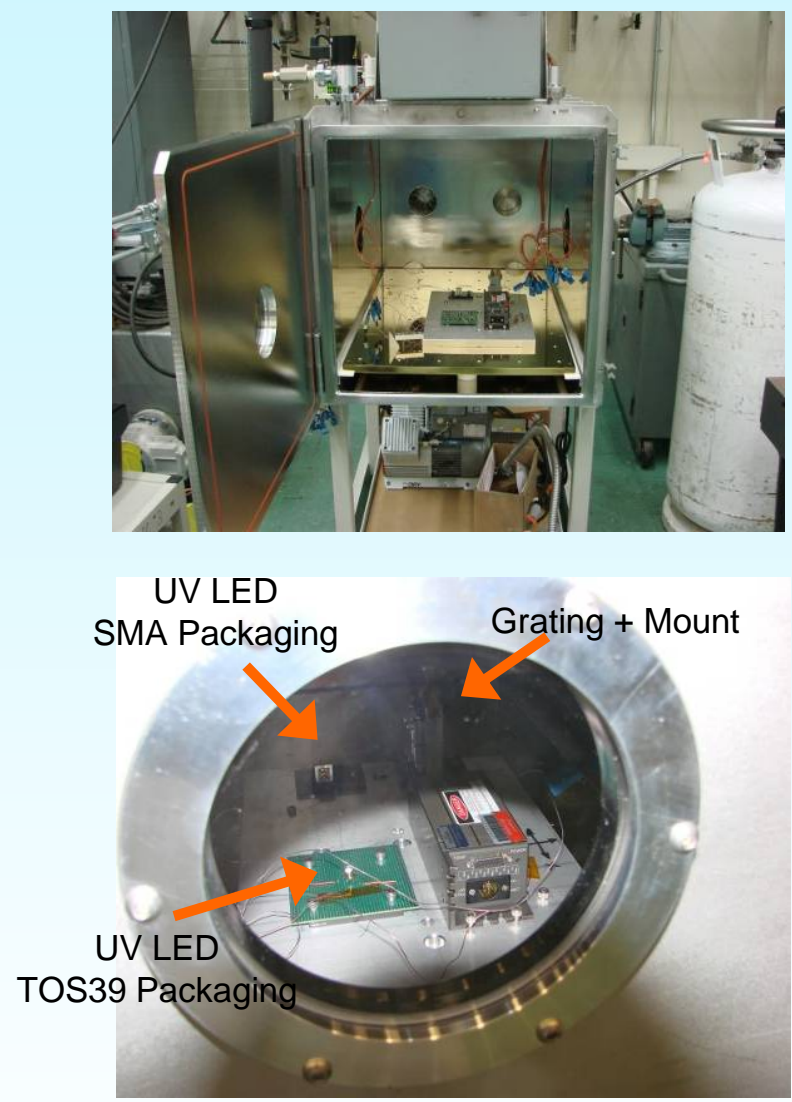

Right column:

Thermal-Vac chamber open-door view (upper), closed door view (lower) -30 +60 C ramp + soak

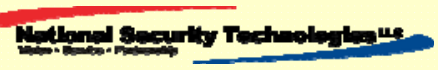




\section{AIGaN UV LED Space Qualification Shake and Bake Tests}

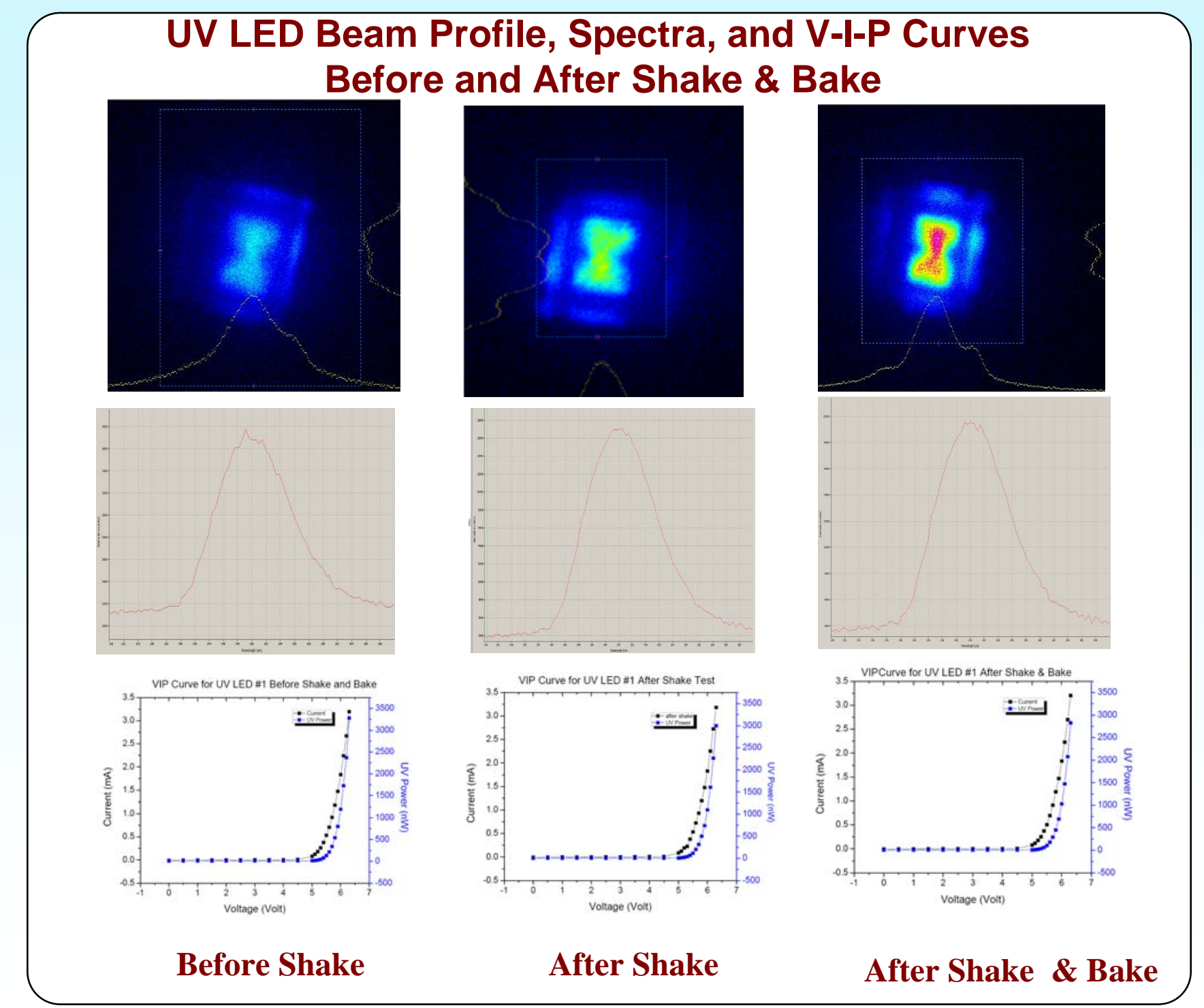




\section{Automated Test Setup 2010}

Karthik Balakrishnan Poster

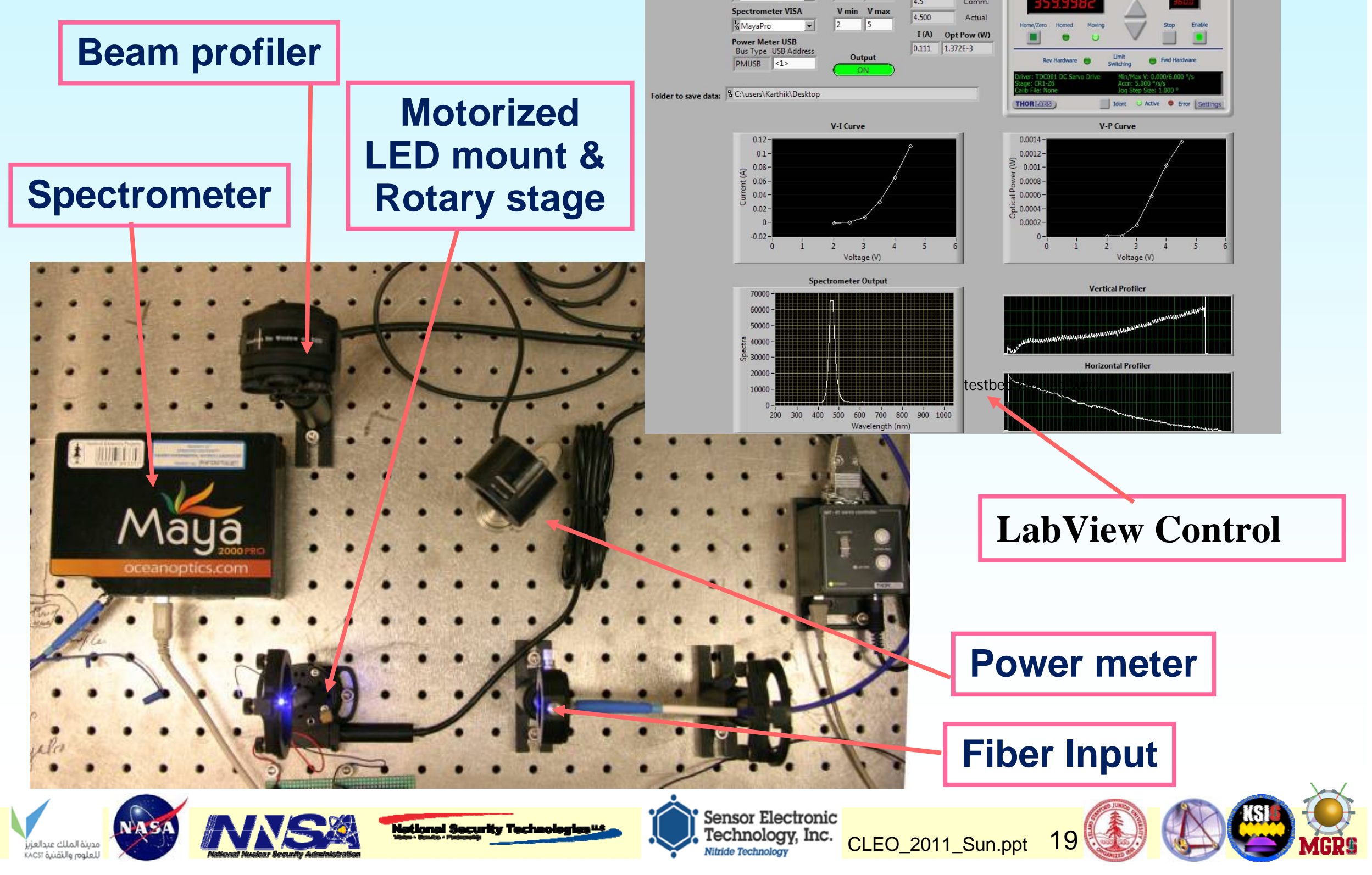




\section{NASA Ames Space Qualification Tests 2010}

- The first uses for the automated testbed was a space qualification test of several UV LEDs at NASA Ames Research Center. The test met the qualification requirements set by MIL-STD-1540E:

- Thermal Vacuum: -34 to $+71 \mathrm{C}$ for 4 cycles

- Vibration: RMS 14g, 6dB above expected profile for 3 minutes/axis

- Thermal cycle at ambient pressure: -34 to $+71 \mathrm{C}$ for 23 cycles
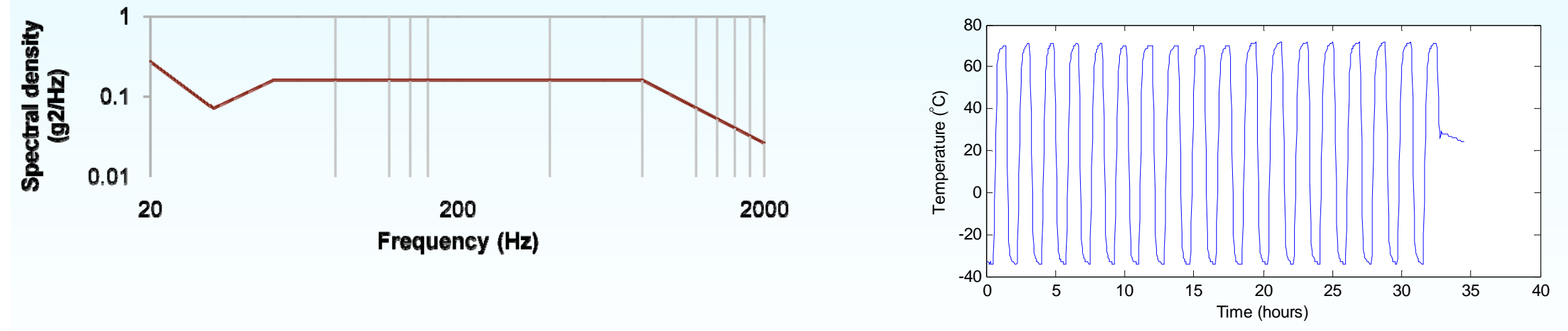


\section{UV LED V-I, I-P Curves, Spectrum Before and After Bake and Shake Tests}

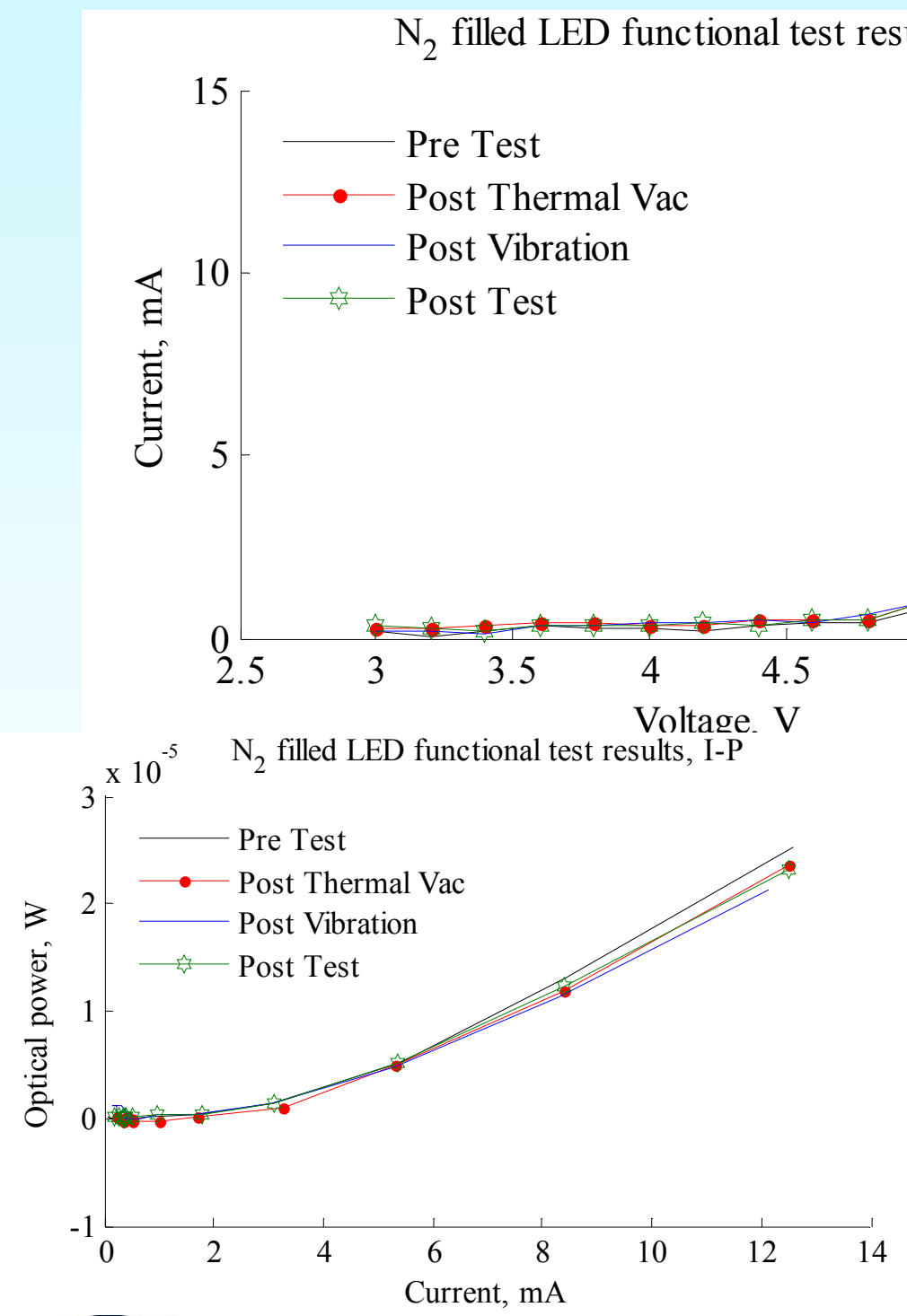

\section{Electrical V-I curves reflects intrinsic semiconductor quality}
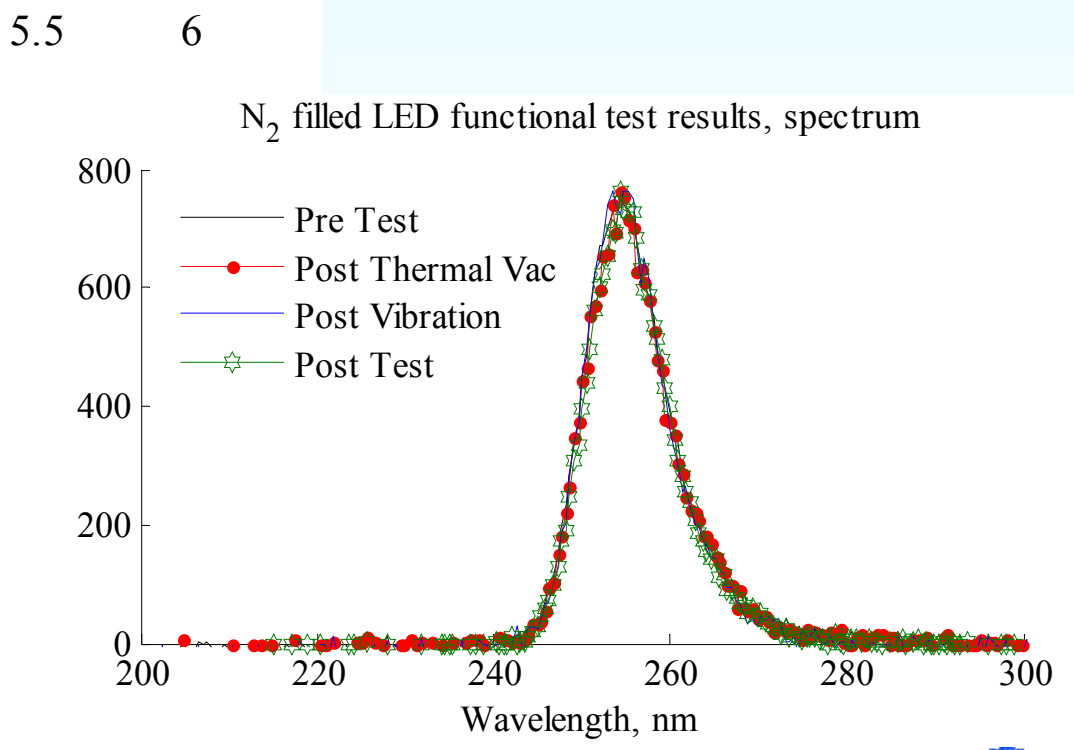


\section{UV LED AC Charge Management}

UV LED Charge Management System

- Light weight, compact size, low power consumption

- Peak wavelength $255 \mathrm{~nm}$, comparable to $\mathrm{Hg}$ line $254 \mathrm{~nm}$

- AC charge management at frequencies out-of signal band (e.g. at cap bridge freq.)

- Long operation time for continuous charge management

Progresses in UV LED space qualification:

- Extreme radiation hardness tested using proton irradiation

- Operational lifetime tests in nitrogen $>26,000$ hours, in vacuum $>30,000$ hours

- Conclusively demonstrated the appropriateness for flying UV LED charge management system
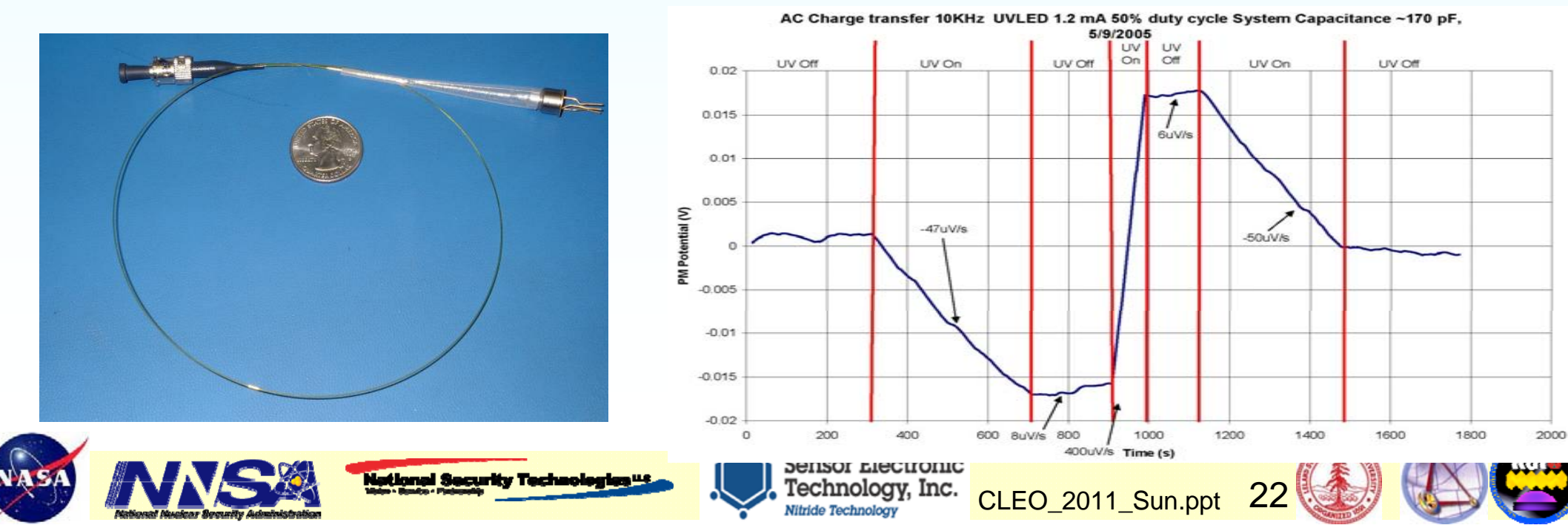


\section{UV LED AC Charge Management}

Institute of Physics Publishing

Classical and Quantum Gravity

Class. Quantum Grav. 23 (2006) S141-S150

doi: 10.1088/0264-9381/23/8/S19

\section{LED deep UV source for charge management of gravitational reference sensors"}

Ke-Xun Sun, Brett Allard, Saps Buchman, Scott Williams and Robert L Byer

Hansen Experimental Physics Laboratory, Stanford University, CA 94305-4085, USA

E-mail: kxsun@stanford.edu

Received 29 September 2005, in final form 3 January 2006

Published 28 March 2006

Online at stacks.iop.org/CQG/23/S141 


\section{UV LED vs. Mercury Lamp Charge Management Systems}

\begin{tabular}{|l|c|c|}
\hline Category & UV LED CMS & Mercury Lamp CMS \\
\hline Electrical Power Consumption & $1 \mathrm{~W}$ & $15 \mathrm{~W}$ \\
\hline EMI & Minimal & Large due to RF excitation \\
\hline Weight & $0.3 \mathrm{~kg}$ & $3.5 \mathrm{~kg}$ \\
\hline Dimension of the CMS system & $10 \mathrm{~cm} \mathrm{x} 8 \mathrm{~cm} \mathrm{x} 3 \mathrm{~cm}$ & $17 \mathrm{~cm} \mathrm{x} 13 \mathrm{~cm} \times 17 \mathrm{~cm}$ \\
\hline UV emission power & $\sim 120 \mu \mathrm{W}$ & $\sim 100 \mu \mathrm{W}$ \\
\hline UV Power at the fiber tip & $\sim 16 \mu \mathrm{W}$ & $\sim 11 \mu \mathrm{W}$ \\
\hline UV Wavelength, central & $257 \mathrm{~nm}$ & $194 \mathrm{~nm} \& 254 \mathrm{~nm}$ \\
\hline UV Wavelength, spread & $12.5 \mathrm{~nm}$ & Doppler Broadening \\
\hline Fast modulation capability & $\begin{array}{c}\text { Yes }- \text { Intensity, pulse } \\
\text { train frequency and } \\
\text { phase, etc. }\end{array}$ & No \\
\hline Charge management method & AC \& DC & DC only \\
\hline Charge management frequency & Out-of signal band & In signal band \\
\hline Equivalent dynamic range & 100,000 & 100 \\
\hline Charge management resolution & high & low \\
\hline Charge management speed & high & low \\
\hline
\end{tabular}




\section{UV LED Charge Management Experimental Setup}

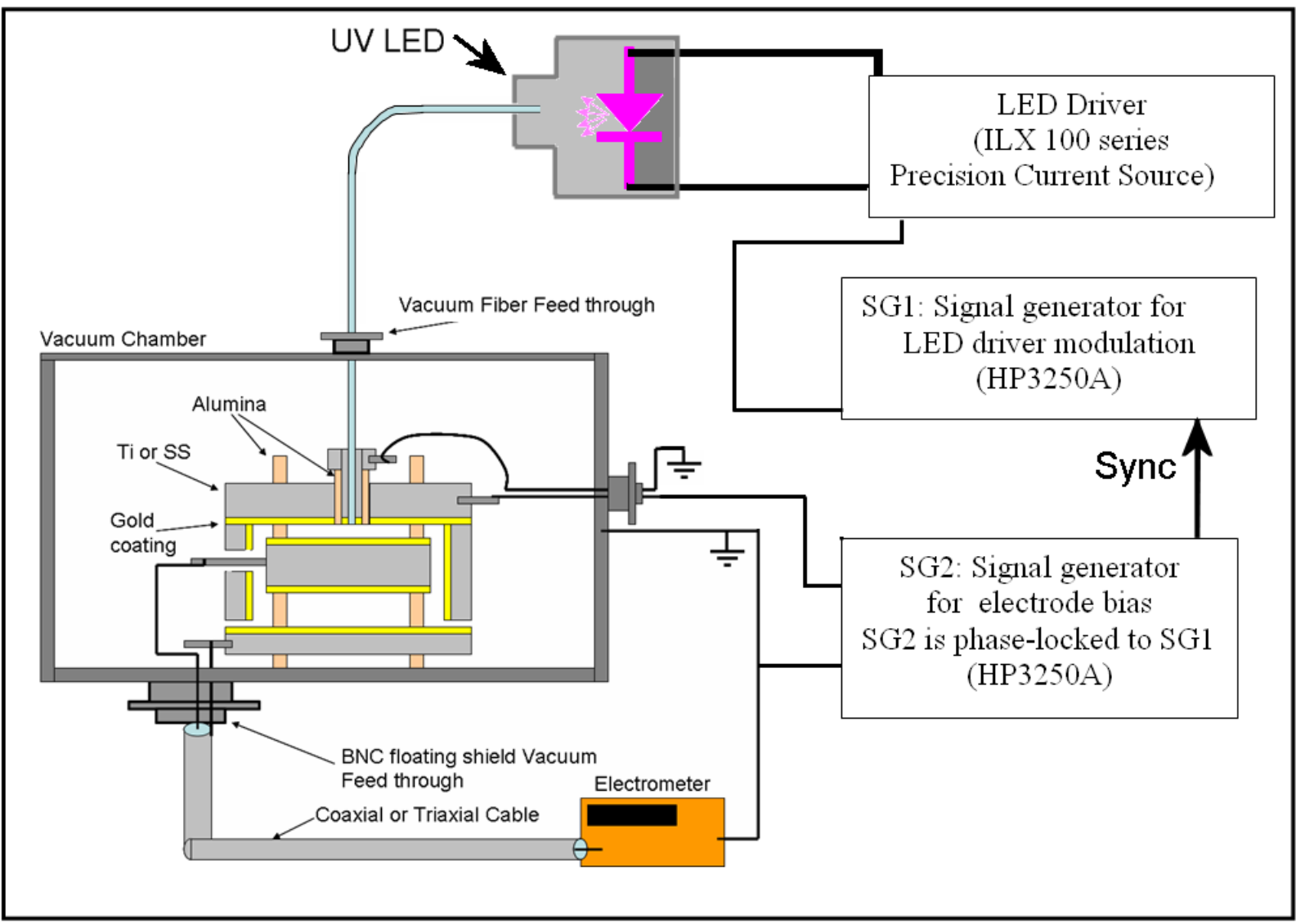

- GP-B heritage

- Au coating on

proof mass and housing to simulate LISA GRS

- Fiber connected UV

LED driven by modulated current source

- Housing electrode modulation phaselocked to UV modulation

- UV light shining on proof mass and reflected onto housing electrode

- Sensitive electrometer to measure the proof mass potential 


\section{Positive Charge Transfer}

\section{UV LED and bias voltage modulated at $1 \mathrm{kHz}$}
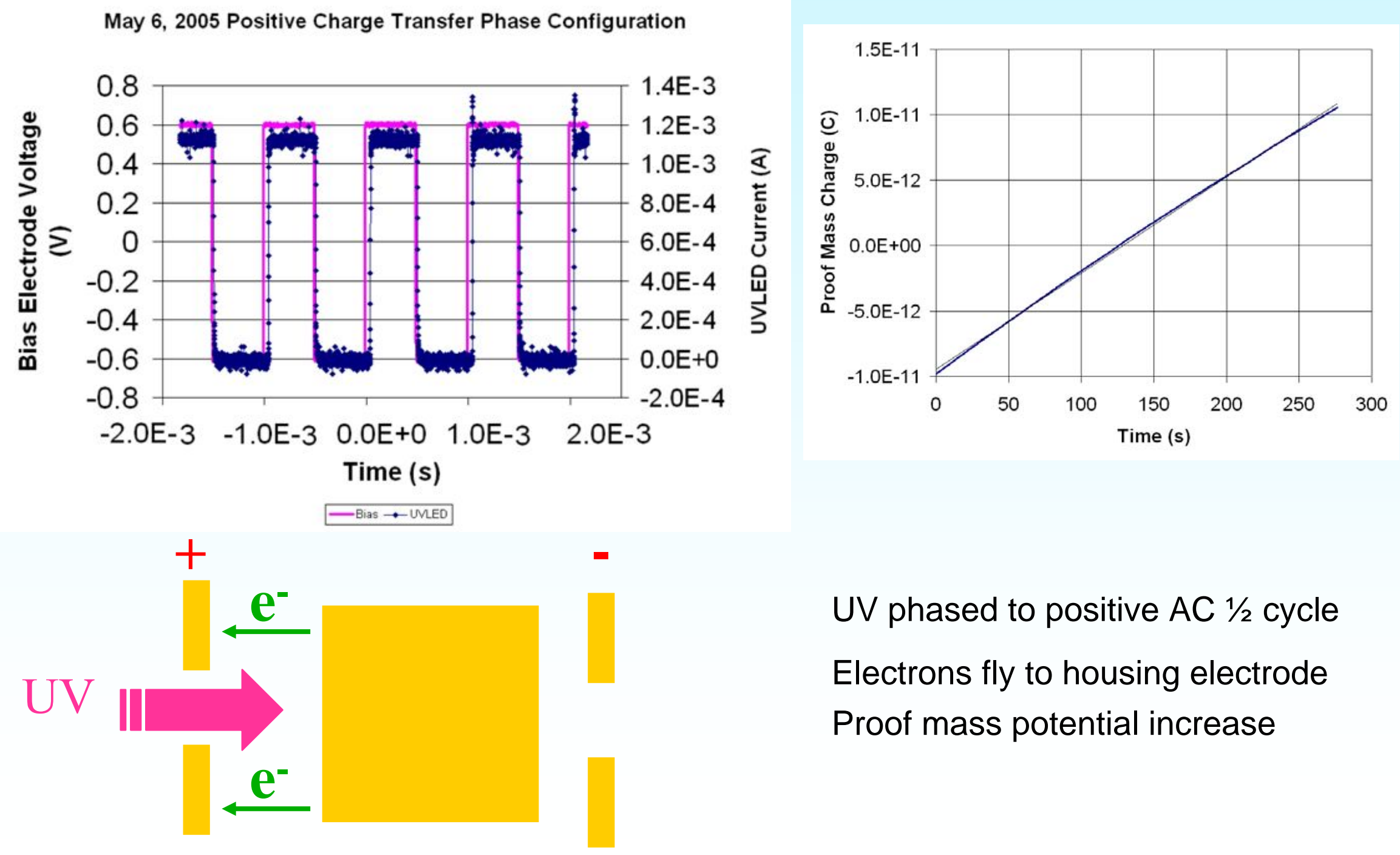

UV phased to positive AC $1 / 2$ cycle Electrons fly to housing electrode Proof mass potential increase 


\section{Negative Charge Transfer}

\section{UV LED and bias voltage modulated at $1 \mathrm{kHz}$}
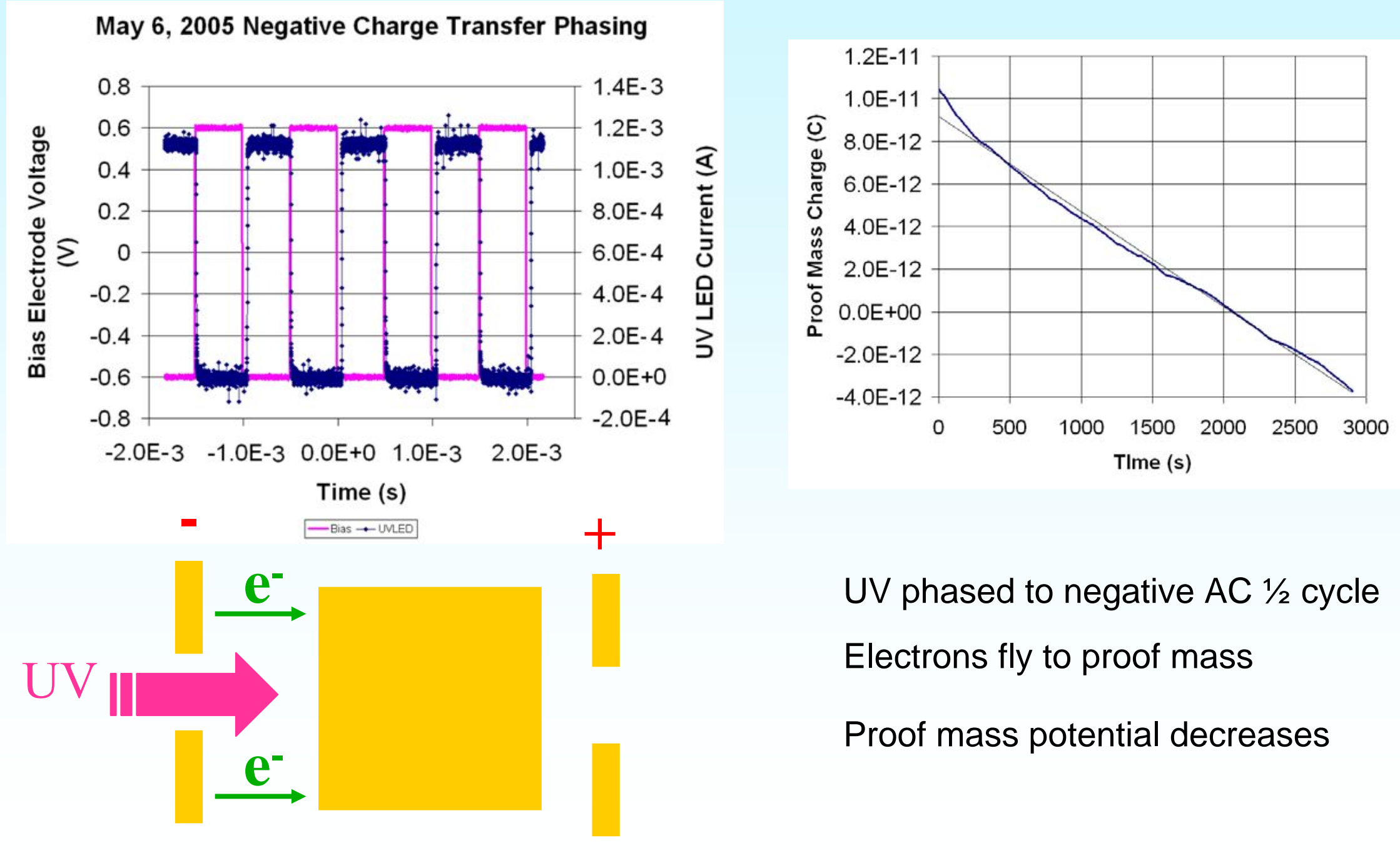

UV phased to negative AC $1 / 2$ cycle Electrons fly to proof mass

Proof mass potential decreases
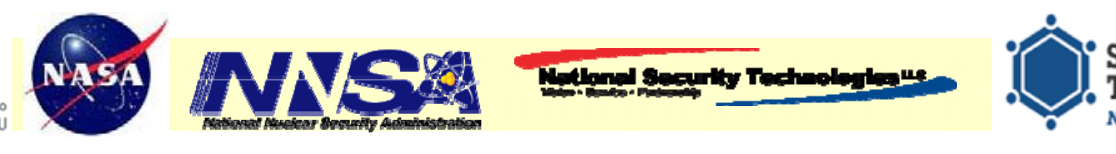


\section{AC Charge Management Shows Promising Characteristics}

\section{UV LED and bias voltage modulated at $10 \mathrm{kHz}$}

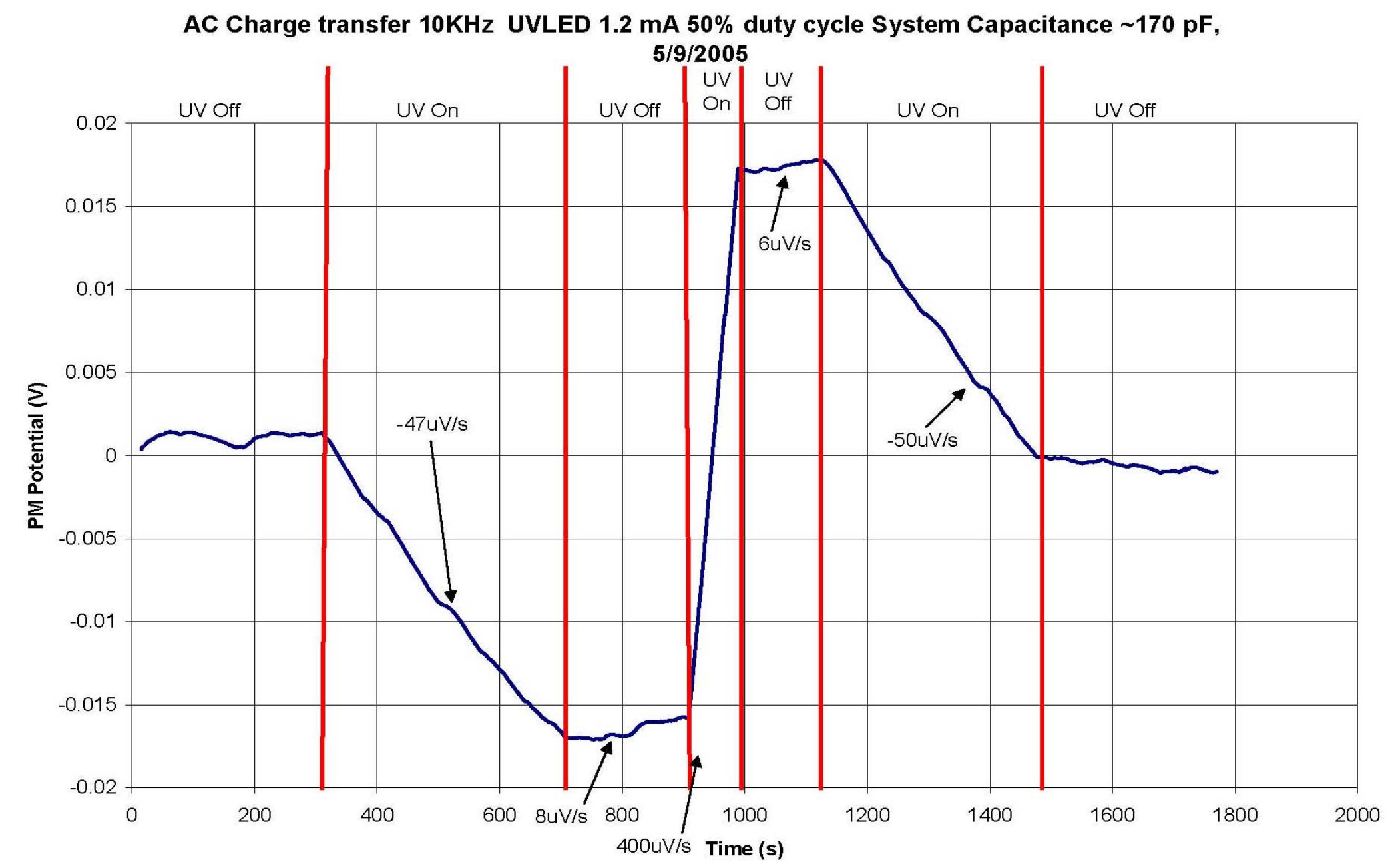




\section{UV LED Space Qualification Environmental Tests}

\begin{tabular}{|c|c|c|}
\hline Category & Test & Performance \\
\hline Lifetime & Operation $\mathrm{N}_{2}$ environment & $\begin{array}{c}>26,000 \text { Hours and on, no UV power } \\
\text { degradation, and significant spectral } \\
\text { shift }\end{array}$ \\
\hline Lifetime & Operation in vacuum $10^{-7}$ tor & $\begin{array}{c}>20,000 \text { Hours and on, no UV power } \\
\text { degradation }\end{array}$ \\
\hline $\begin{array}{c}\text { Radiation } \\
\text { Hardness }\end{array}$ & Proton Irradiation & $\begin{array}{c}2 \times 10^{12} \text { protons/cm } \text { irradiation, no } \\
\text { power and spectra shift }\end{array}$ \\
\hline $\begin{array}{c}\text { Shake tests (I) } \\
\text { (May 2009) }\end{array}$ & $\begin{array}{c}\mathrm{X}, \mathrm{Y}, \mathrm{Z} \text { 3g \& 7g spread spectrum shake } \\
\text { (Delta II Vehicle) }\end{array}$ & $\begin{array}{c}\text { No significant changes in power, } \\
\text { spectral, and V-I-P characteristics }\end{array}$ \\
\hline $\begin{array}{c}\text { Bake tests (I) } \\
\text { (May 2009) }\end{array}$ & $\begin{array}{c}\text { Thermal Vac 10-6 tor } \\
\text { 2 Temperature cycles } \\
\text { including soak }\end{array}$ & $\begin{array}{c}\text { No significant changes in power, } \\
\text { spectral, and V-I-P characteristics }\end{array}$ \\
\hline $\begin{array}{c}\text { Shake tests (II) } \\
\text { (June 2010) }\end{array}$ & $\begin{array}{c}\mathrm{X}, \mathrm{Y}, \mathrm{Z} \text { 14g spread spectrum shake } \\
\text { (GEVS Vehicle) }\end{array}$ & $\begin{array}{c}\text { No significant changes in power, } \\
\text { spectral, and V-I-P characteristics }\end{array}$ \\
\hline $\begin{array}{c}\text { Bake tests (II) } \\
\text { (June 2010) }\end{array}$ & $\begin{array}{c}\text { Thermal Vac 10-6 tor } \\
\text { 23 Temperature cycles } \\
\text { including soak }\end{array}$ & $\begin{array}{c}\text { No significant changes in power, } \\
\text { spectral, and V-I-P characteristics }\end{array}$ \\
\hline
\end{tabular}




\section{Modular Gravitational Reference Sensor}
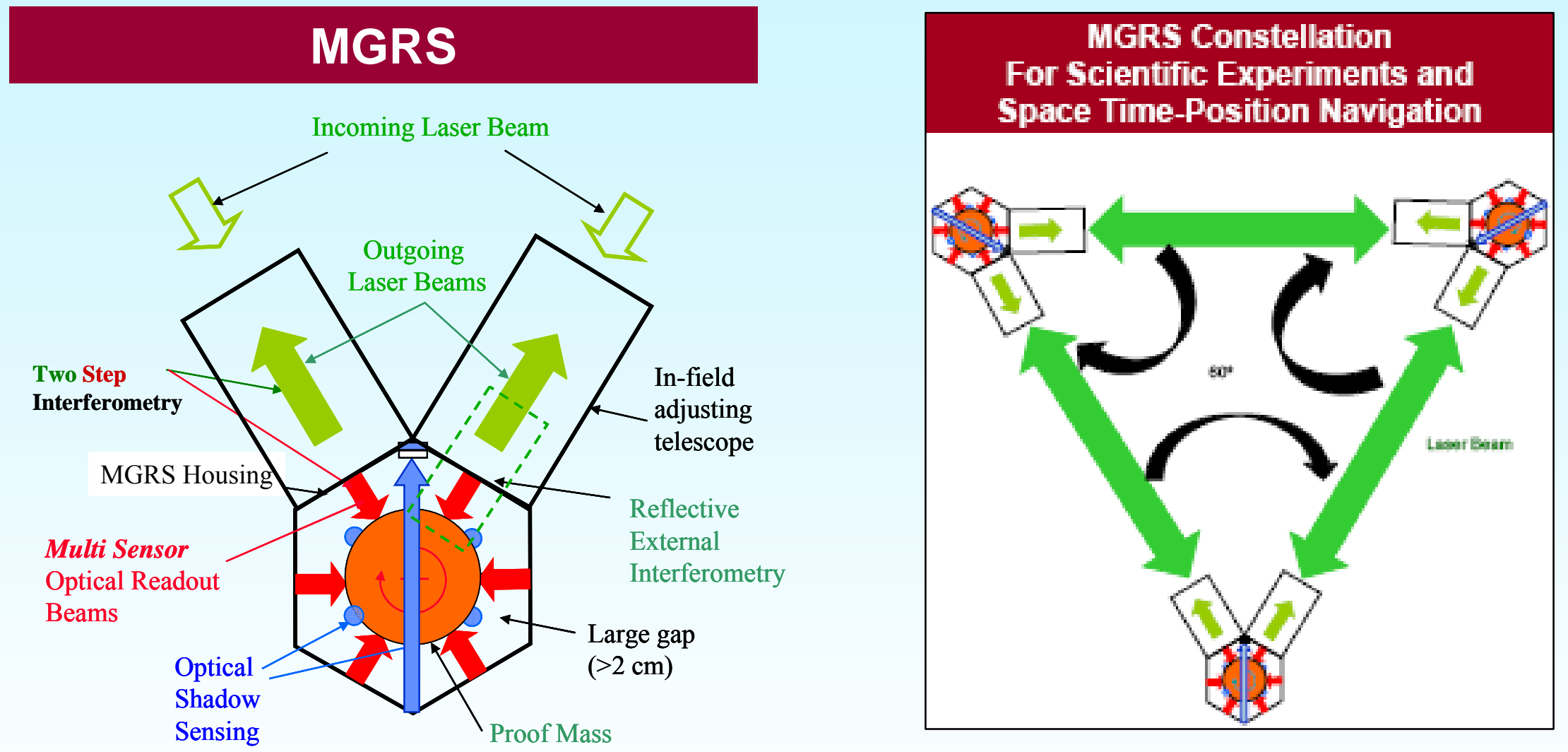

MGRS is the next generation space gravitational reference sensor

Sun, Allen, Buchman, DeBra, Byer, LISA $5^{\text {th }}$ Symposium ESTEC 2004, CQG (22) 2005 S287-S296 


\section{MGRS Small Satellite Series UV LED is the First Mission}

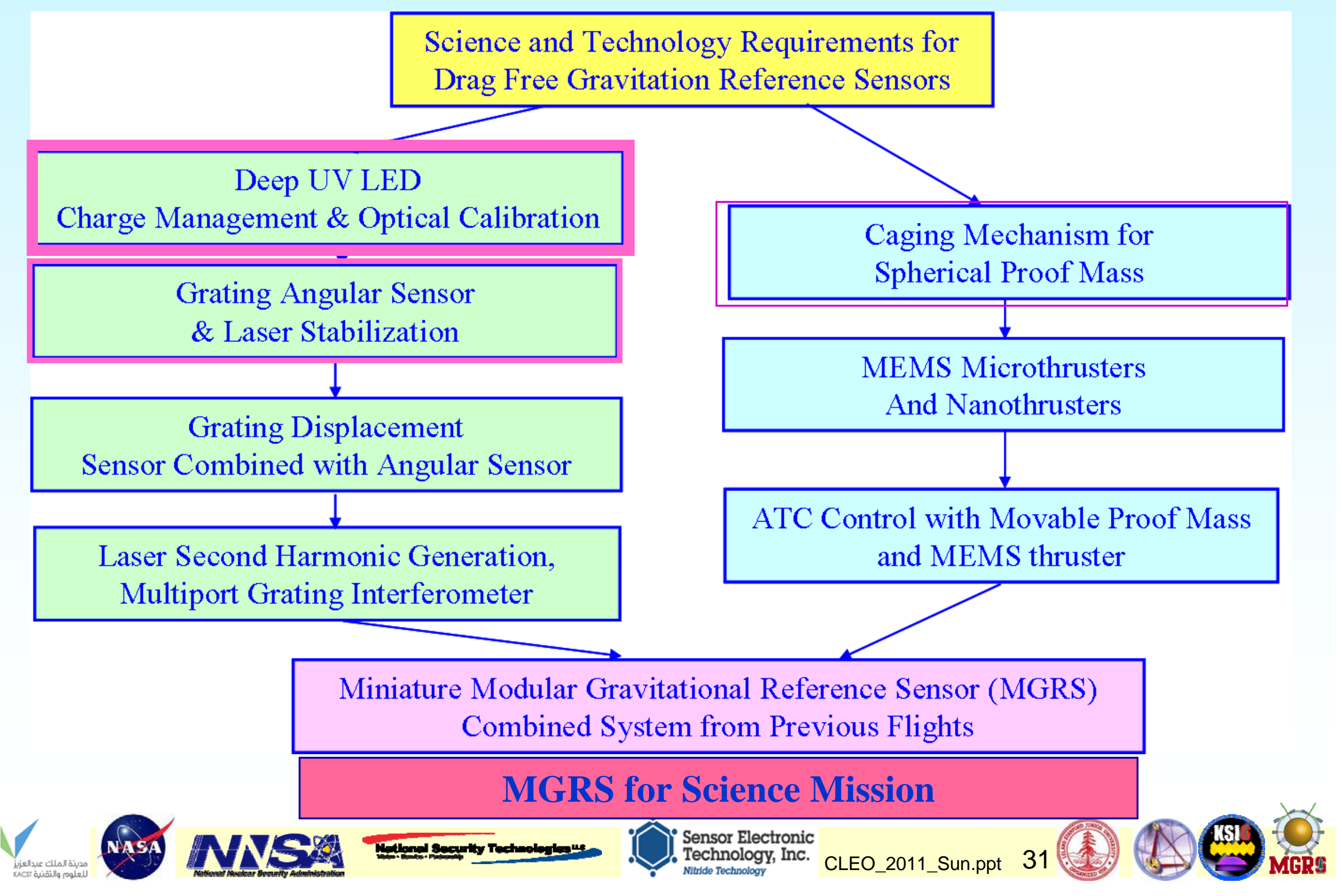




\section{UV LED Satellite Payload Design Evolution}

- 2005 Experiment

- 2006 Design

- Geared to nanosat (Cube Sat)

- Payload 9x9x18 cm $\mathrm{cm}^{3}=1458$ $\mathrm{cm}^{3}$

- 2009 Design

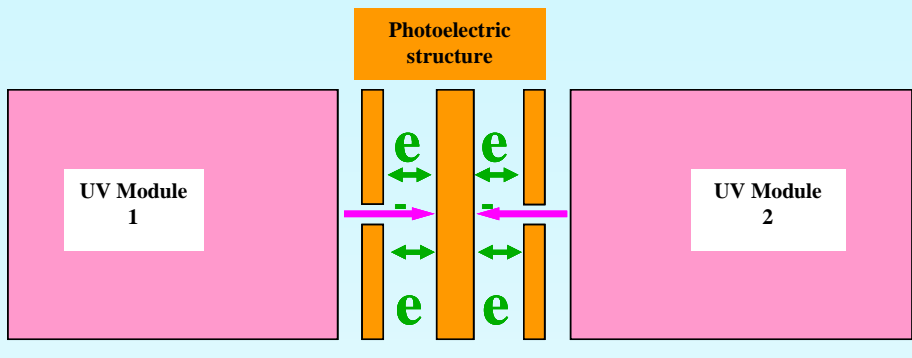

Uppē Deck Top View

- Resized to allow COTS parts

- Reshaped to hexagon for MGRS constellation configuration

- 2010 Design

- KACST larger satellite allowing more UV LED testing

- Full charge management system

- Total payload dimension $30 \times 30 \times 20 \mathrm{~cm}^{3}=18,000 \mathrm{~cm}^{3}$

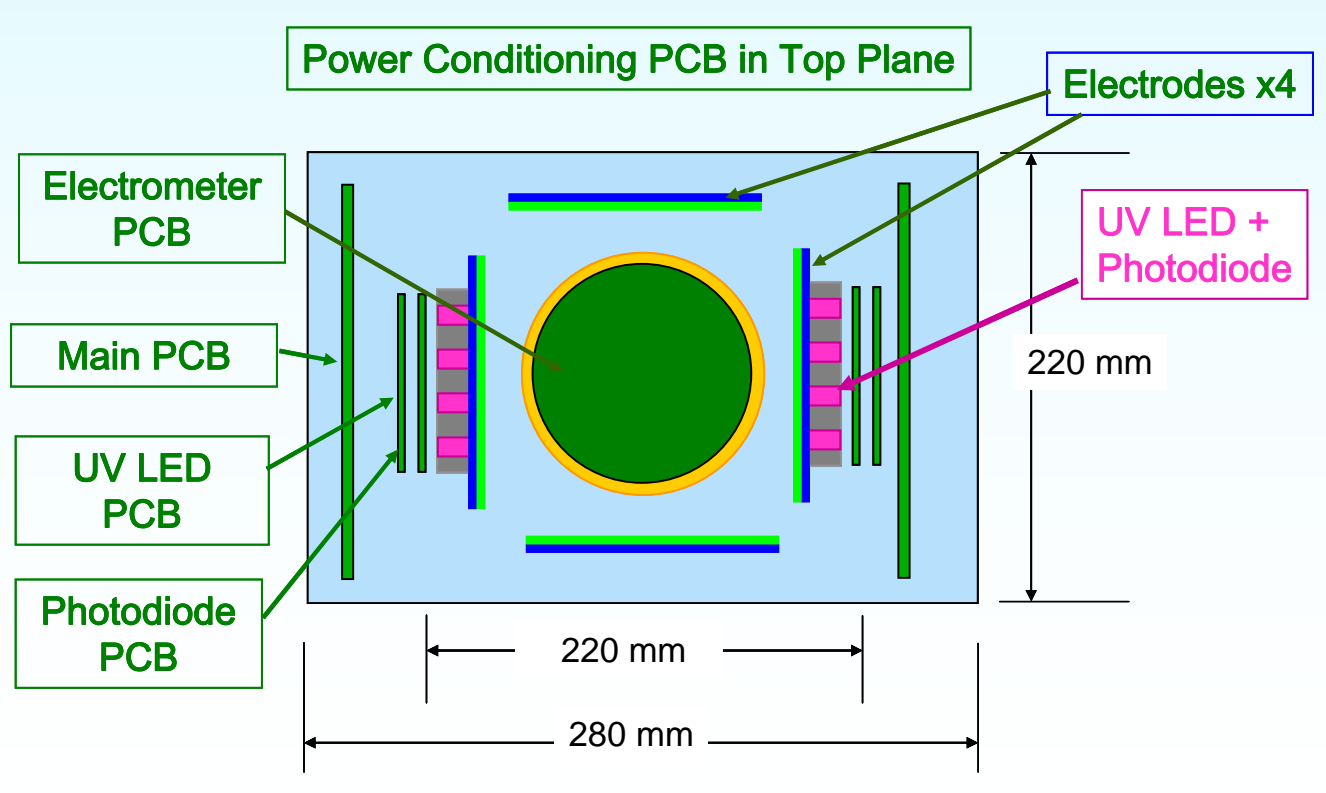




\section{UV LED Main Payload}

Option 1:

Overall Dimensions (mm) $(270 \times 190 \times 138)$

4 Boards (182x138)

4 Flexible Boards (30x 84.77)

2 Boards $(65 \times 80)$
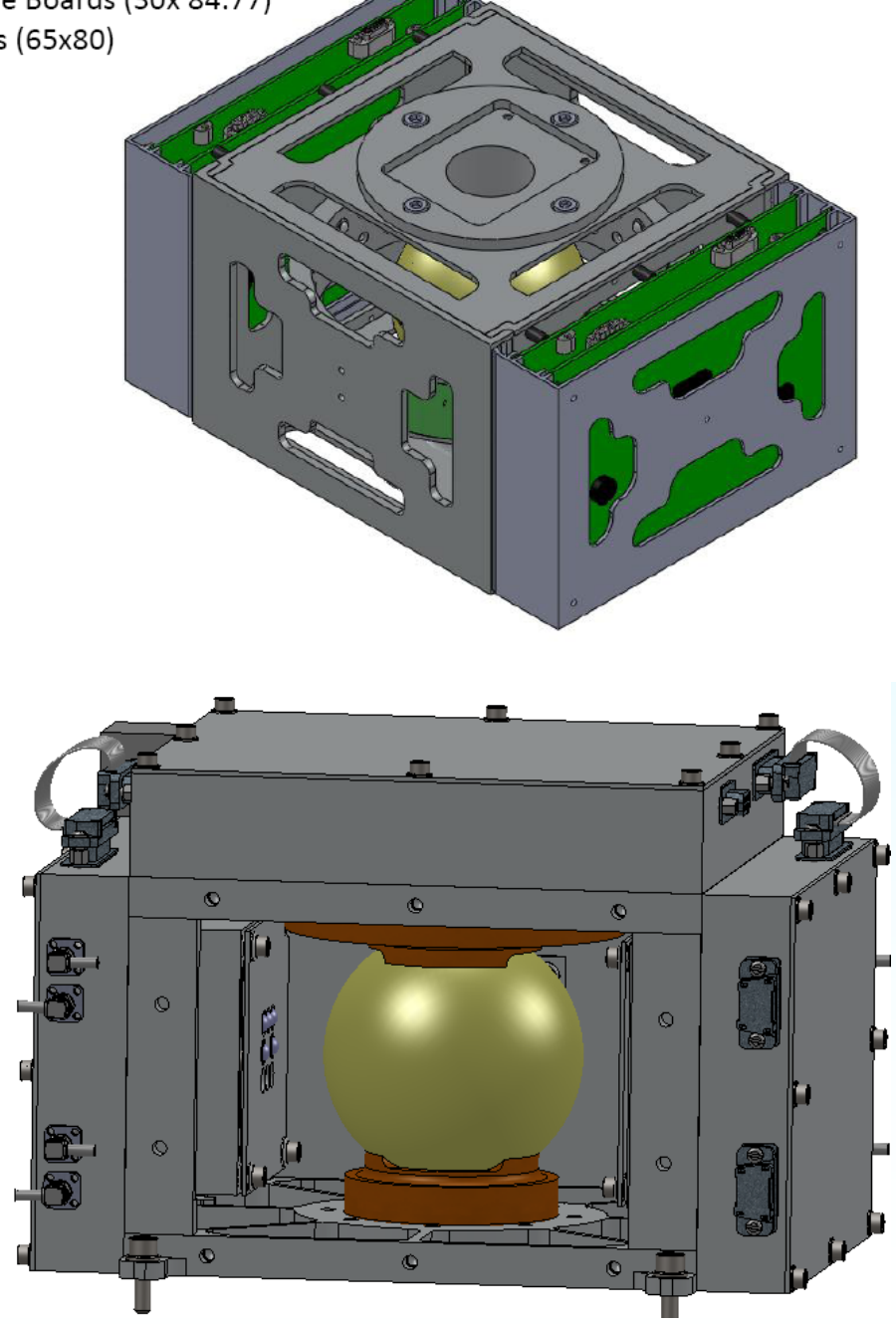
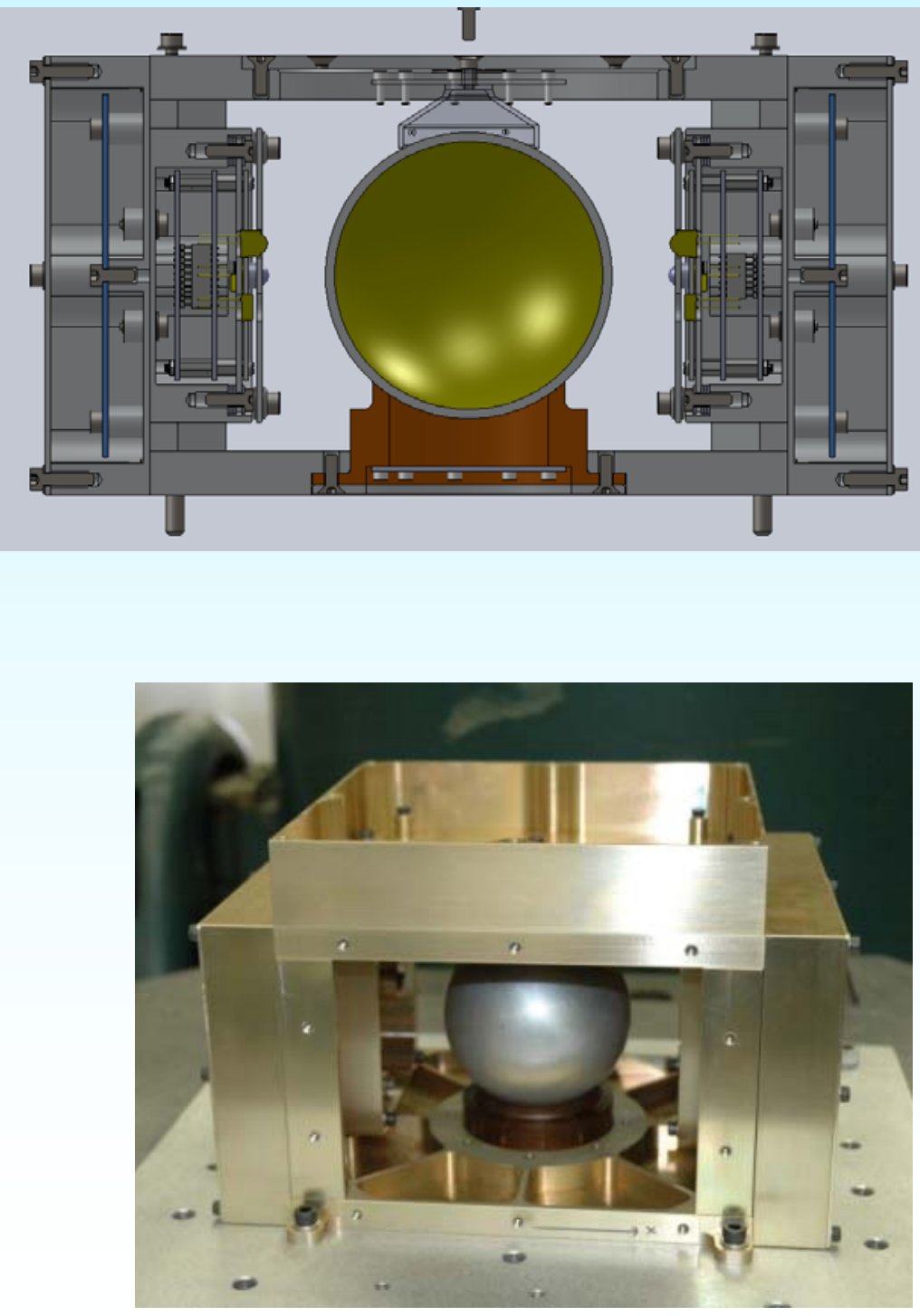


\section{UV LED Optical Configuration and Modeling}
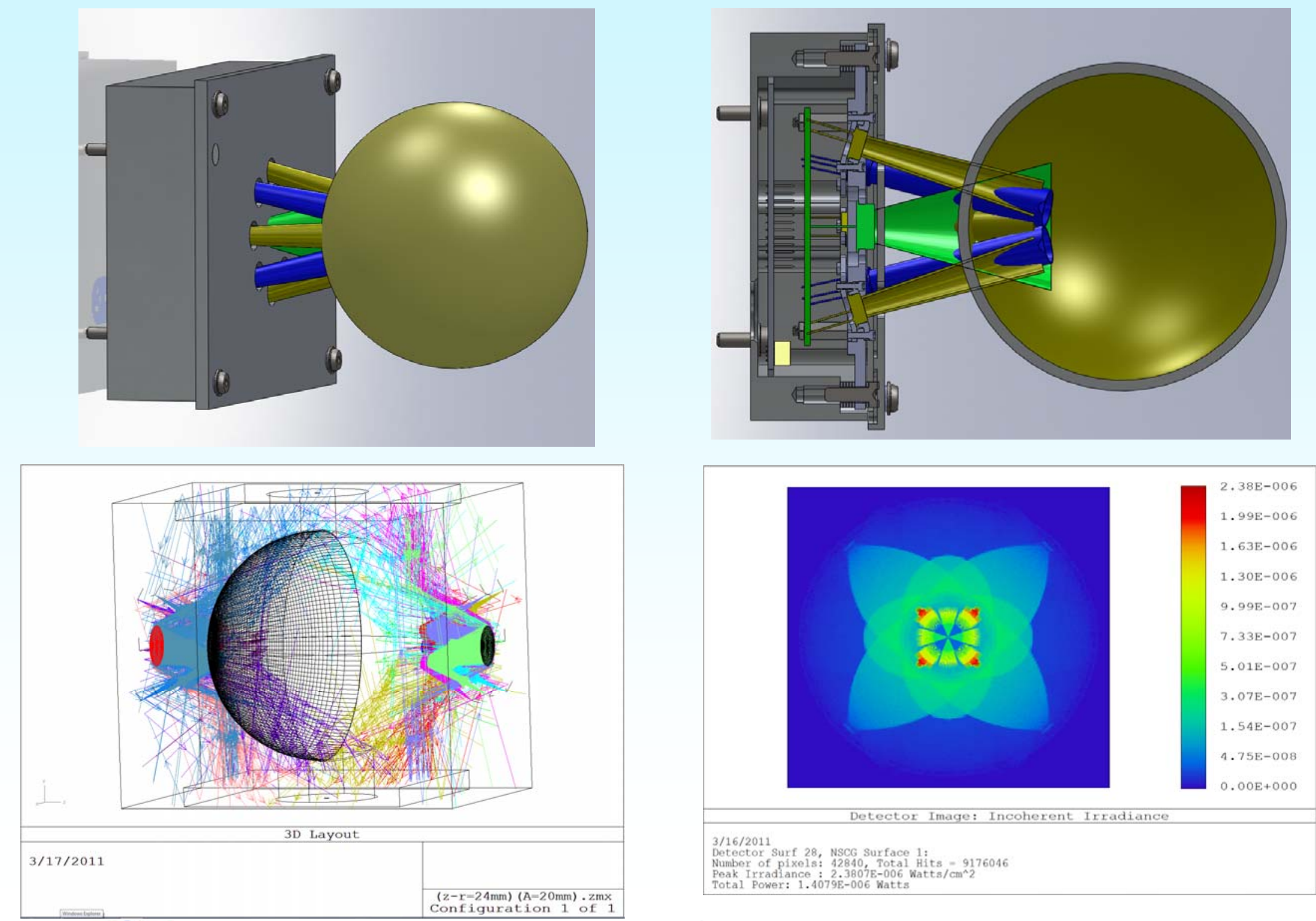

$\checkmark$ 에요

1)

34 


\section{UV LED AC Charge Management Experiment}

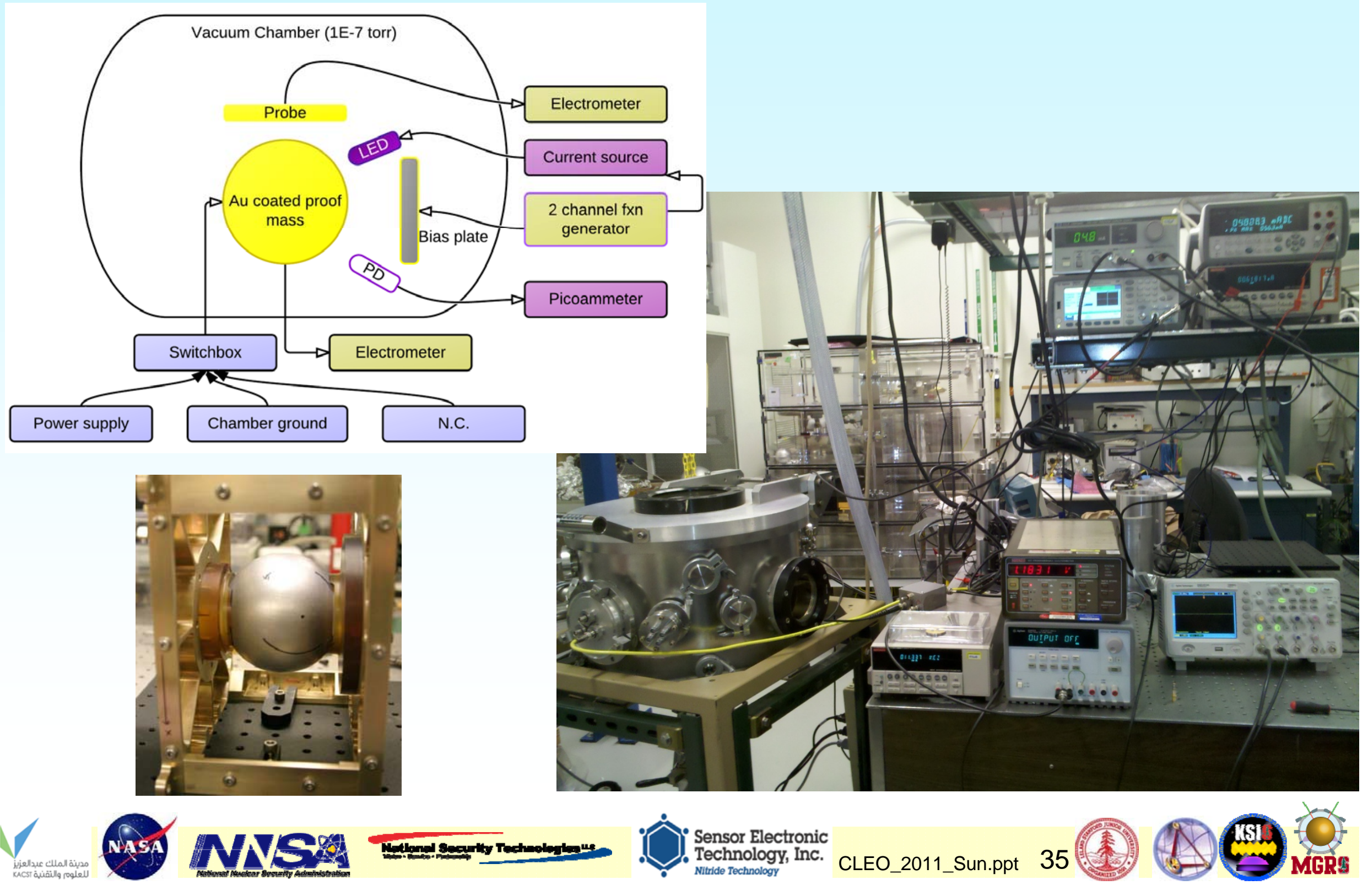




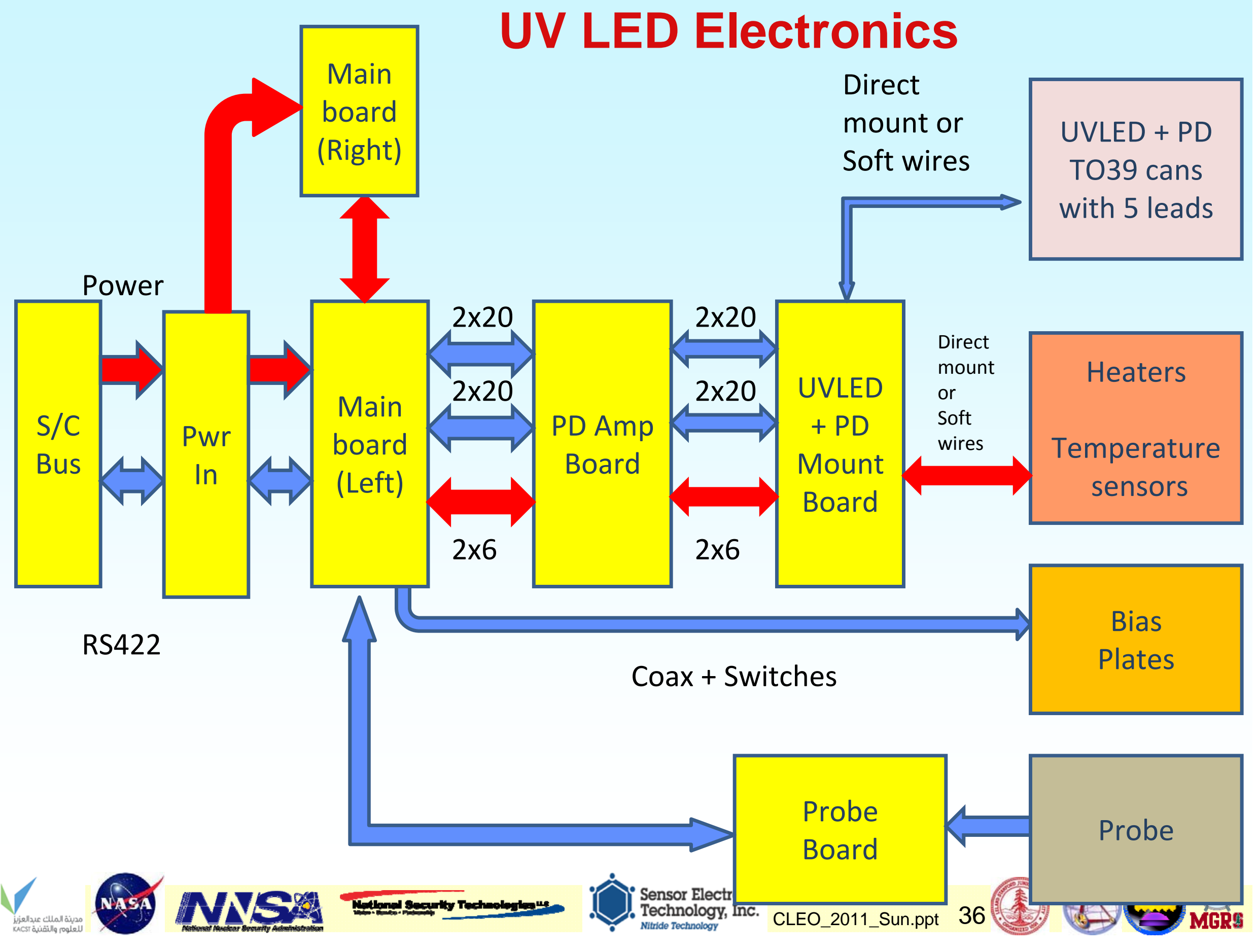




\section{UV LED AC Charge Management Works in the Spherical Proof Mass, Large Gap Configuration}

Single LED driven at $6.4 \mathrm{~mA}(\sim 10 \mu \mathrm{W}), 3.0 \mathrm{~V}_{\mathrm{PP}}$ bias, $100 \mathrm{~Hz}$
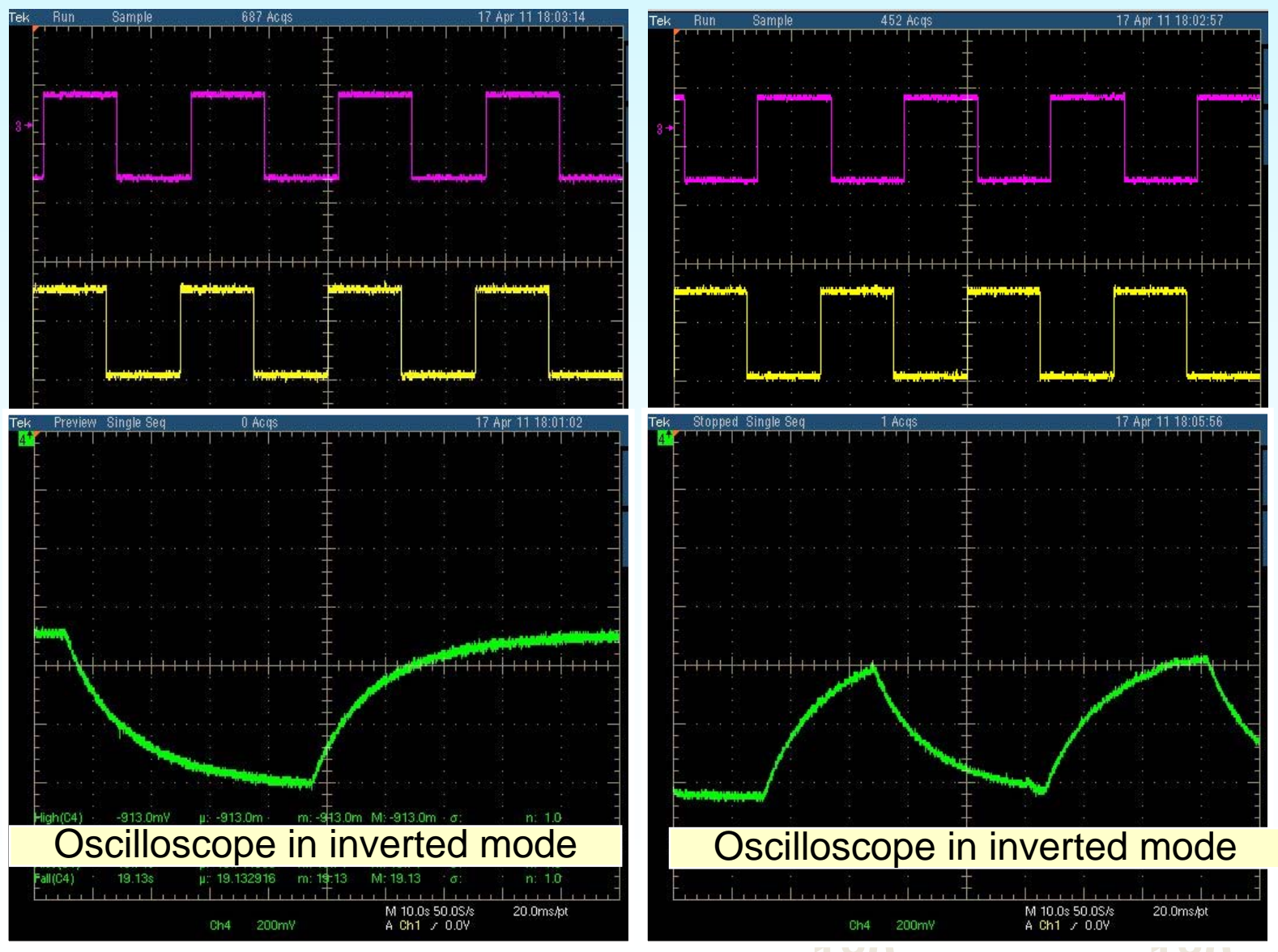

Oscilloscope in inverted mode

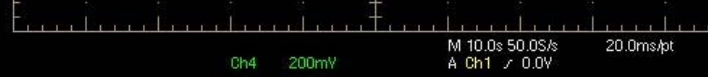




\section{UV LED Schedule and Program}

- Launch Date: July 1, 2012

- Payload completion: September 2011

- Ames, KACST, Stanford team working together until mission completion

- Integrated mission team

- No middle hand over from engineering model to flight model

- Expert review instead of program review

- The first in MGRS Series and STAR Series

- Demonstration a key MGRS/LISA technology

- STAR series risk mitigation

- DECIGO collaboration 


\section{UV LED Satellite Work Breakdown Structure}

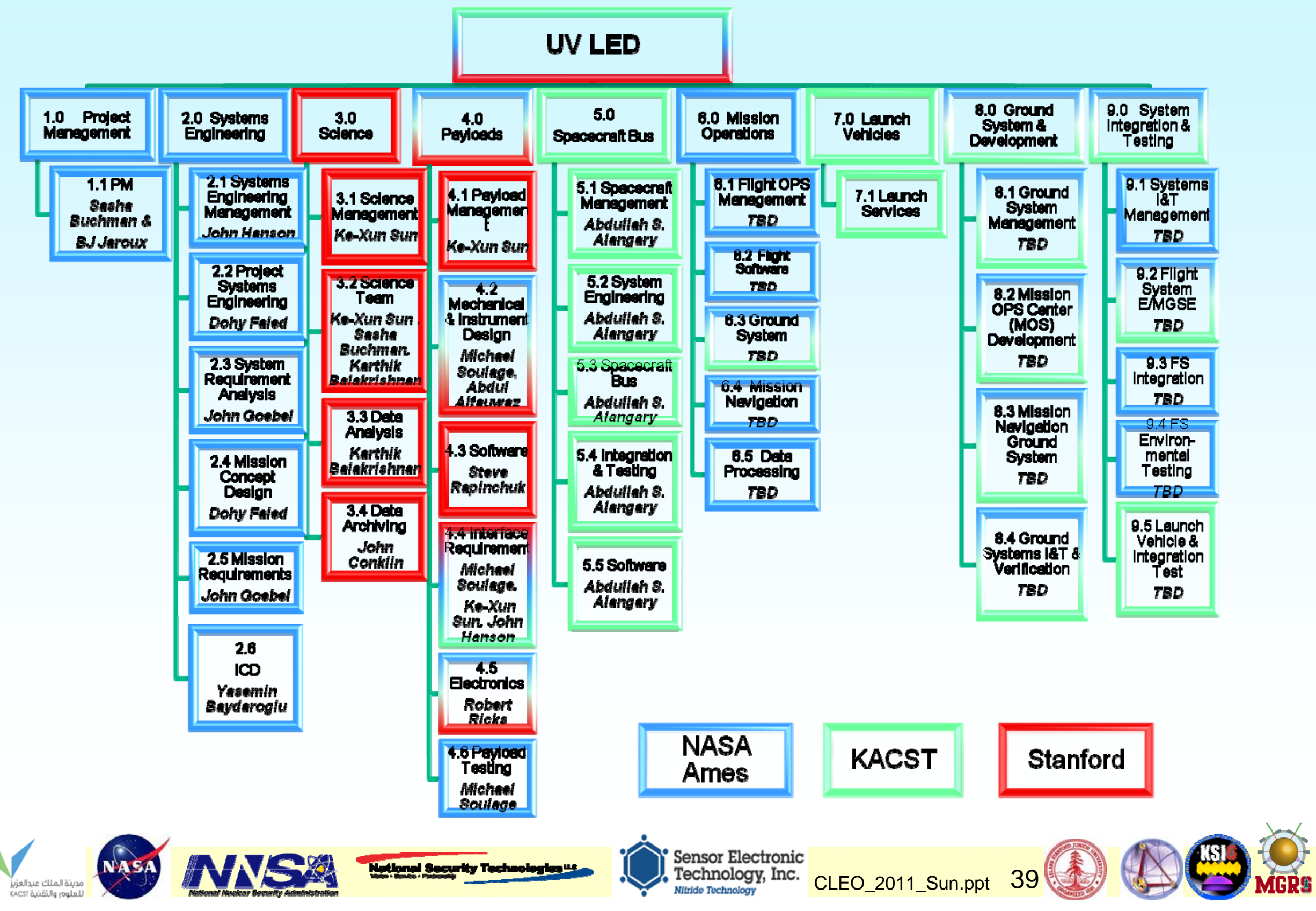




\section{Conclusions and Outlook}

- AIGaN UV LED and Photodiodes

- Extreme radiation hardness

- Environmental robustness

- Long operation lifetime

- UV LED AC charge management

- Cubic PM GRS structure

- Spherical MGRS structure

- UV LED Satellite

- Successful payload design and demonstration

- Launch in $2^{\text {nd }}$ half 2012

- Outlook

- The first MGRS small satellite series

- AIGaN imaging devices and system

- AIGaN optoelectronic devices have broad impacts to

- Space exploration

- High energy density physics

This work was done by National Security Technologies, LLC, is under Contract No. DE-AC52-06NA25946 with the U.S. Department of Energy. DOE/NV/25946--1221 